(C) 2022, The Authors. Published by Elsevier Inc. and Fass Inc. on behalf of the American Dairy Science Association ${ }^{\circledR}$. This is an open access article under the CC BY license (http://creativecommons.org/licenses/by/4.0/).

\title{
Comparison of microbiota of recycled manure solids and straw bedding used in dairy farms in eastern Canada
}

\author{
Jessika Beauchemin, ${ }^{1,2,3 *}$ ๑ Annie Fréchette, ${ }^{1,3} \odot$ William Thériault, ${ }^{1,2} \oplus$ Simon Dufour, ${ }^{1,3} \oplus$

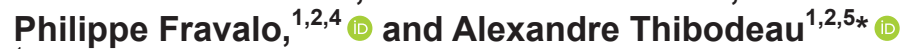 \\ ${ }^{1}$ Département de Pathologie et Microbiologie, Faculté de Médecine Vétérinaire, Université de Montréal, Saint-Hyacinthe, \\ Québec J2S 2M2 Canada \\ ${ }^{2}$ Chaire de recherche en salubrité des viandes (CRSV), Université de Montréal, Saint-Hyacinthe, Québec J2S 2M2 Canada \\ ${ }^{3}$ Regroupement FRQ-NT Op+Lait, Saint-Hyacinthe, Québec J2S 2M2 Canada \\ ${ }^{4}$ Pole Agro-alimentaire, Conservatoire national des arts et métiers, Le Cnam, 22440 Ploufragan, France \\ ${ }^{5}$ Centre de recherche en infectiologie porcine et avicole (CRIPA-FRQNT), Saint-Hyacinthe, Québec J2S 2M2 Canada
}

\begin{abstract}
Recycled manure solids (RMS) bedding is an alternative bedding option that is growing in popularity on Canadian dairy farms. However, the microbiological characteristics and production of RMS bedding are poorly documented under on-farm conditions in eastern Canada. This bedding could support the presence of pathogens and could have an effect on cow and human health. The aim of this study was to describe the RMS microbiota when used under dairy cows and compare it with straw bedding. Unused and used bedding from 27 RMS and 61 straw-bedded dairy farms were collected and compared using $16 \mathrm{~S}$ amplicon sequencing, bacterial counts, and Salmonella spp. and Listeria monocytogenes detection. Microbiota composition of unused RMS and unused straw were different. After use, both bedding microbiota were similar in their bacterial composition, structure, and diversity. Unused RMS generally contained higher bacterial counts than did unused straw, except for Klebsiella spp. counts. Salmonella spp. and $L$. monocytogenes were more frequently detected in unused RMS (Salmonella spp.: 11\%; L. monocytogenes: 30\%), than in unused straw (Salmonella spp.: 0\%; L. monocytogenes: 11\%). Finally, 2 RMS production systems (extraction of the liquid fraction followed by maturation in an opened or enclosed container vs. in a heap) did not influence the microbiota richness and bacteria distribution ( $\alpha$-diversity), but did influence the microbiota structure ( $\beta$-diversity). In conclusion, animal and human pathogens were found in greater numbers and
\end{abstract}

Received March 25, 2021.

Accepted September 2, 2021.

*Corresponding authors: jessika.beauchemin@hotmail.com and alexandre.thibodeau@umontreal.ca more frequently in unused RMS than unused straw, and this could eventually affect dairy cow or human health. Key words: dairy cow, bedding, recycled manure solids, straw, microbiota

\section{INTRODUCTION}

Various beddings are used on dairy farms in eastern Canada. In this region, straw is the most common option chosen by producers (Dufour et al., 2010). The annual cost of straw, however, reached Can $\$ 542$ per cow per year in eastern Canada (Ontario and Québec) in 2019 (Godbout et al., 2019). Therefore, there is a growing interest in alternative bedding options such as recycled manure solids (RMS) bedding.

Recycled manure solids bedding can be produced on the farm. Usually, a fraction of the liquid contained in the manure is extracted using a screw or roller press. The liquid fraction of the manure can then be used as an organic fertilizer in fields (Godbout et al., 2019). The remaining solid fraction can be used immediately as bedding, but it will often be further processed using various maturation methods, such as static heap, turned windrow, or a drum composter (Leach et al., 2015; Fournel et al., 2019a,b). During maturation, the material's temperature should increase due to bacterial fermentation, ensuring a reduction in the total bacterial concentration (Carroll and Jasper, 1978; Leach et al., 2015). On larger farms, anaerobic digesters can also be used to produced RMS (Leach et al., 2015). Currently, there is no legislation guiding RMS production or use in Canada. This situation has led to a large variation in RMS production methods, as each farmer is using their own RMS production system, often homemade.

Some studies have identified various benefits associated with RMS, such as increased comfort, reduced hock lesions, and improved cleanliness of dairy cows (Green et al., 2014; Bradley et al., 2018). On the other 
hand, other studies have observed larger undesirable bacterial populations, such as populations of Klebsiella spp., Escherichia coli, Streptococcus spp., and Staphylococcus spp. (Cole and Hogan, 2016; Rowbotham and Ruegg, 2016a). These bacteria can cause mastitis and affect milk production and quality (Cole and Hogan, 2016; Rowbotham and Ruegg, 2016a). A study has reported an association between the use of RMS and mastitis caused by Klebsiella spp. (Leach et al., 2015). Moreover, in general, the relative number of bacteria present on teat skin is associated with bedding and, thus could possibly alter the incidence of clinical mastitis (Rowbotham and Ruegg, 2016b).

Zoonotic pathogen transmission is also a concern regarding use of RMS. Lasprilla-Mantilla et al. (2019) demonstrated that Cryptosporidium spp. (mainly Cryptosporidium parvum, Cryptosporidium andersoni, and Cryptosporidium meleagridis), zoonotic microorganisms, were not controlled by the RMS production methods used on eastern Canadian dairy farms. They found that these pathogens were more frequently recovered in adult cows' feces from RMS farms (85\%) compared with farms using straw (49\%). They suggested that use of RMS could promote within-herd persistence of these pathogenic agents. This situation should be better documented for other pathogens such as Salmonella and Listeria monocytogenes, 2 relevant zoonotic pathogens in milk production.

Several studies have compared the bacterial load of RMS bedding with those of other bedding types in the United States, United Kingdom, and Ontario (Godden et al., 2008; Bradley et al., 2018; Robles et al., 2020). Knowledge is still scarce on the evolution of RMS microbiota before and after use in our northern conditions. The possibility of maintaining or even promoting pathogenic and zoonotic microorganisms on a farm is poorly documented. The objective of this study was to describe the RMS microbiota in eastern Canadian dairy farms according to production method using both culture-dependent (i.e., bacterial counts) and independent (high-throughput sequencing) methods and to compare the results with those of straw bedding. A second objective was to investigate the presence of 2 zoonotic agents, Salmonella spp. and L. monocytogenes, in RMS and straw. Because these pathogens are zoonotic, they could affect the dairy cow health and could be transmissible to humans via direct contact with the animals or bedding, or indirect contact with contaminated milk products (Nightingale et al., 2005; Hunt et al., 2012; Holschbach and Peek, 2018; OIE, 2019). We hypothesized that RMS usage would further reduce the microbiological quality of the bedding, potentially compromising cow and human health.

\section{MATERIALS AND METHODS}

\section{Farm Recruitment and Sampling}

All procedures were approved by the Animal Care and Use Committee of the Faculty of Veterinary Medicine of the Université de Montréal (Protocol 17-Reach-1886). Study design was an observational cross-sectional study and was described thoroughly in Lasprilla-Mantilla et al. (2019) and Gagnon et al. (2020). Briefly, a list of RMS farms located within $250 \mathrm{~km}$ of the Faculty of Veterinary Medicine of the Université de Montréal (Saint-Hyacinthe, QC, Canada) in the provinces of Québec and Ontario was constructed by contacting veterinarians, via social networks, and through equipment dealers. The owners of all the identified RMS farms were contacted and asked to participate in the study. The selection criteria for RMS farms to be included were as follows: (1) the farms needed to use RMS as the primary bedding type for lactating cows; and (2) RMS needed to have been used for at least 6 mo prior the start of the study. Several farms using straw were selected for comparison and were recruited in the same area and with similar criteria as for RMS farms to reach a total number of farms of approximately 90. All of these farms used straw materials originating from dried stalks from grain production. None of the selected farms used poor quality hay as bedding materials. Due to budget constraints and other parameters, such as availability of RMS farms and to increase statistical power, a 2:1 ratio of straw to RMS was used for farm selection. Straw-bedded farms were recruited with the help of the eastern Canadian DHIA (Valacta Inc., Sainte-Anne-de-Bellevue, QC, Canada). Thus, strawbedded farms were all conducting regular DHI milk control analyses, whereas this was not necessarily the case for RMS-bedded herds.

Procedures used for producing RMS were recorded. It is important to note that the RMS farms produced RMS using different protocols, involving some bedding maturation (Figure 1). Both used and unused RMS or straw samples were collected by the research team. Unused bedding was sampled directly from the distribution equipment before being put in place, and used bedding was sampled after at the end of the use cycle (which varied from one farm to another; Table 1), just before replacement. Used bedding was sampled randomly from the back one-third surface of 5 stalls in each farm, avoiding manure piles (Animal and Health Diagnostic Center, 2020). Bedding samples (from the 5 different stalls; total of $300 \mathrm{~g}$ ) were then mixed in a plastic bag, and a 50-g fraction was immediately transferred to a $50-\mathrm{mL}$ conical tube that was plunged into 


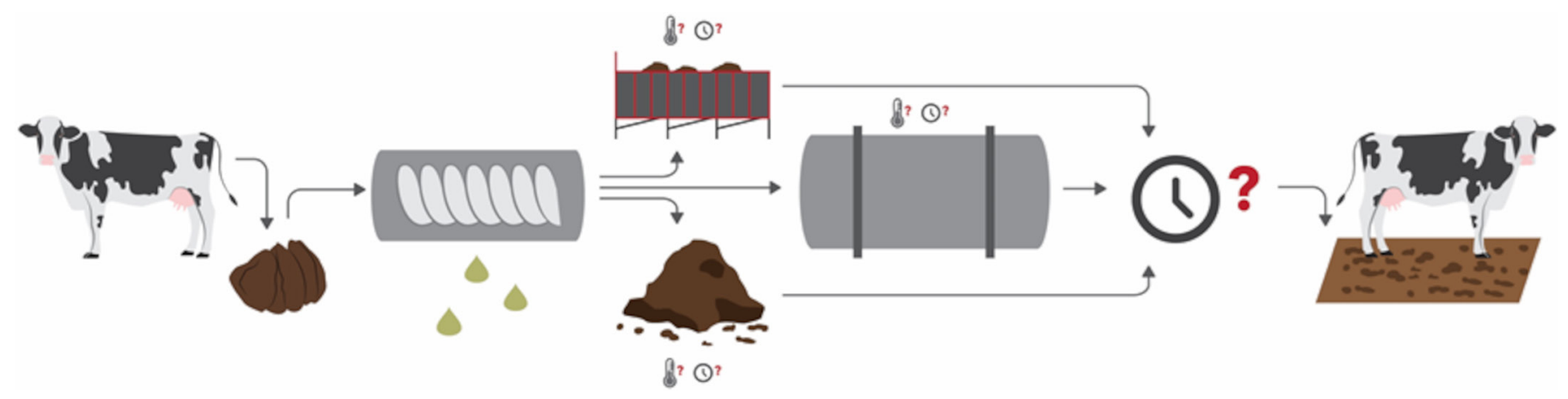

Figure 1. General recycled manure solids production in eastern Canadian dairy farms. A fraction of the liquid contained in manure is extracted using a screw or roller press. The remaining solid fraction can then be used immediately as bedding, but it will often be further processed using various maturation steps. The final product is then used in stalls. (Figure from Aida Minguez Menendez; Université de Montréal).

liquid nitrogen and kept at $-80^{\circ} \mathrm{C}$ until DNA extraction analyses. The remaining bedding materials contained in the plastic bag were transported on ice to the laboratory, were stored at $-20^{\circ} \mathrm{C}$, and processed within 3 mo for classical microbiological analyses. The quantity of liquid nitrogen and ice was adapted depending on transport time to preserve sample integrity. Thus, on each farm, 2 unused (1 for DNA extraction and 1 for classical microbiology) and 2 used bedding samples were available for analyses.

\section{Microbiota Analysis by 16 S Amplicon Sequencing}

DNA Extraction. One gram of bedding sample conserved at $-80^{\circ} \mathrm{C}$ (used and unused RMS and straw) was added to $5 \mathrm{~mL}$ of PBS (Oxoid Canada) in a filter bag and homogenized using a stomacher. The PBS solution, containing the bacteria detached from the bedding material, was kept. This process was repeated 3 times for each sample to maximize bacterial recovery. The total recovered PBS solution (approximately $15 \mathrm{~mL}$ ) was centrifuged at $4^{\circ} \mathrm{C}$ for $20 \mathrm{~min}$ at $4,700 \times$ $g$ (Thermo Sorvall Legend X1, Thermo Fisher), and the pellet used for DNA extraction. Genomic DNA extraction of all samples was performed with the Qiagen DNeasy PowerSoil Kit (Qiagen), as recommended by the manufacturer. Negative control (water) and positive control (ZymoBIOMICS Microbial Community Standard; Zymo Research) were used. The DNA concentration was measured with an Invitrogen Qubit dsDNA HS assay kit (Thermo Fisher Scientific). Each DNA sample was standardized to a concentration of $2 \mathrm{ng} / \mu \mathrm{L}$. After standardization, the DNA was stored at $-20^{\circ} \mathrm{C}$ until PCR amplification.

PCR Amplification. A 291 bp fragment of the V4 region of the $16 \mathrm{~S}$ rRNA gene from the total extracted DNA was amplified. The PCR negative controls (wa- ter) and PCR positive controls (ZimoBIOMICS Microbial Community DNA Standard; Zymo Research) were used. The amplification was conducted according to the manufacturer. The primers 515FP1-CS1F (5'-TACGGTAGCAGAGACTTGGTCTGGACTACHVGGGTWTCTAAT-3') and 806RP1-CS2R (5'-ACACTGACGACATGGTTCTACAGTGCCAGCMGCCGCGGTAA-3') were used (Caporaso et al., 2012). The PCR master mix for one reaction volume of $30 \mu \mathrm{L}$ contained Invitrogen Platinum SuperFi DNA polymerase $(1 \times \mathrm{Su}-$ perFi buffer, $1 \times$ enhancers and $0.2 \mathrm{U} / \mu \mathrm{L}$ SuperFi Taq polymerase; Thermo Fisher Scientific), $0.2 \mathrm{~m} M$ dNTPs, $0.6 \mu M$ each primer, and $0.4 \mathrm{mg} / \mathrm{mL}$ BSA. To each well, $6 \mu \mathrm{L}$ of DNA $(2 \mathrm{ng} / \mu \mathrm{L})$ was added and amplified according to the following program: initial denaturation at $95^{\circ} \mathrm{C}$ for $5 \mathrm{~min}, 23$ cycles $(30 \mathrm{~s}$ of denaturation at $95^{\circ} \mathrm{C}, 30 \mathrm{~s}$ annealing at $55^{\circ} \mathrm{C}$, and an elongation of 180 s at $72^{\circ} \mathrm{C}$ ), and a final elongation of $10 \mathrm{~min}$ at $72^{\circ} \mathrm{C}$ (Thibodeau et al., 2017). The PCR amplification was confirmed by electrophoresis on $2 \%$ agarose gel.

Sequencing and Bioinformatics Analysis. The PCR products (samples, negative DNA extraction controls, negative PCR control, positive DNA extraction controls, and positive PCR control) were sent to Génome Québec Inc. (Montréal, QC, Canada) for Illumina MiSeq $2 \times 250 \mathrm{pb}$ sequencing. Reads were received in FastQ files from the sequencing center. Mothur version 1.43.0 was used for reads cleaning following the online MiSeq SOP (https://mothur.org/wiki/miseq_sop/, accessed February 2020; Kozich et al., 2013). Briefly, for each sample, complementary sets of reads were combined. Sequences that were too long or contained ambiguities were discarded. Reads were aligned and classified using SILVA's database version 132 (Thibodeau et al., 2017) and similar sequences regrouped with the deblur precluster method. Chimeras were removed, and reads were clustered into operational taxonomic units 


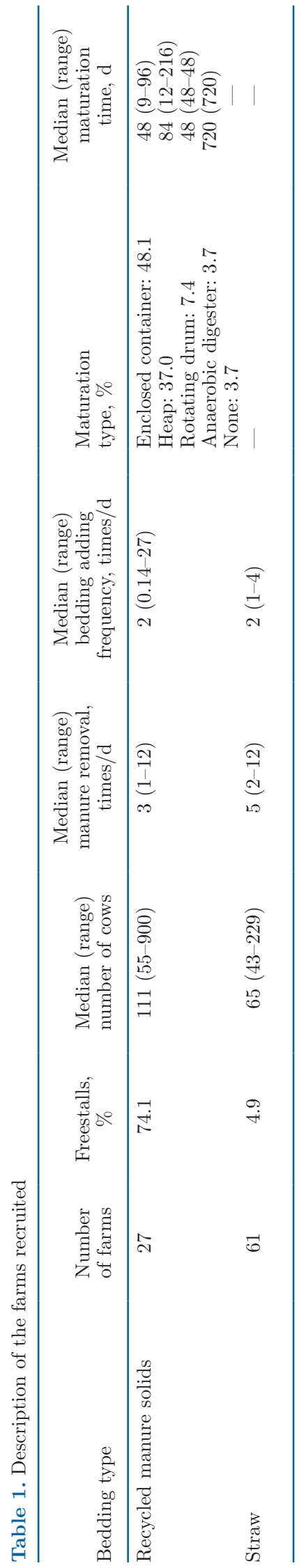

(OTU) with the "unique" option (Larivière-Gauthier et al., 2017; Thibodeau et al., 2017). For analysis, reads were subsampled to the lowest numbers of reads found in a single sample. The raw sequences are accessible on the National Center for Biotechnology Information sequence read archive platform (https://www.ncbi.nlm .nih.gov/sra/PRJNA734942).

\section{Bacterial Analyses}

Investigating the Presence of Salmonella spp. Detection was made using $25 \mathrm{~g}$ of each bedding sample stored at $-20^{\circ} \mathrm{C}$ (used and unused RMS and straw). The methodology was based on a modified version of the International Organization for Standardization standard 6579:2002(E) and MFHPB-20 procedures previously used in our laboratory (Lebel et al., 2017). Briefly, Salmonella spp. detection was performed using a preincubation in buffered peptone water (Biokar diagnostic) at $37^{\circ} \mathrm{C}$ for 18 to $24 \mathrm{~h}$. Tetrathionate brilliant green bile broth (TBG; BD Difco) and modified semi-solid Rappaport-Vassiliadis (MSRV) agar medium (Biokar Diagnostics) with novobiocin $(20 \mathrm{mg} / \mathrm{L}$; Sigma-Aldrich) were used in parallel for selective enrichment. We inoculated TBG and MSRV, respectively, with $1 \mathrm{~mL}$ and 3 spots of $100 \mu \mathrm{L}$ of the pre-enrichment. They were incubated for 24 to $48 \mathrm{~h}$ at $42^{\circ} \mathrm{C}$. Isolation was performed on BG sulfa agar brilliant green medium (BGS; BD Difco) inoculated with a needle of the MSRV migration zone extremity and in parallel on xylose lysine deoxycholate (XLD) medium (Biokar Diagnostics) inoculated with the incubated TBG. Both BGS and XLD were incubated at $37{ }^{\circ} \mathrm{C}$ for $24 \mathrm{~h}$ (Lebel et al., 2017).

One typical colony per plate (from BGS and XLD) was selected for confirmation using triple sugar iron (TSI) medium (BD Difco), urea agar medium (BD BBL), and seroagglutination using Salmonella O antiserum poly A-I factors 1 to 16 (Fisher Scientific). Confirmed colonies were sent to the Animal Health Laboratory of the Québec Ministry of Agriculture, Fisheries, and Food (MAPAQ, Saint-Hyacinthe, QC, Canada) for serogrouping (Supplemental Table S1; https://github .com/CRSV).

Investigating the Presence of $L$. monocytogenes. Detection was made using $10 \mathrm{~g}$ of each sample (used and unused RMS and straw). This detection was performed with a modified version of the Rapid'L.mono method of Bio-Rad Laboratories (Bio-Rad, 2014). A primary enrichment was first performed in modified UVM 1 broth (Biokar Diagnostics) for $48 \mathrm{~h}$ at $30^{\circ} \mathrm{C}$, followed by a secondary enriched $(100 \mu \mathrm{L}$ of UVM 1$)$ in Fraser broth $\left(24 \mathrm{~h}\right.$ at $37^{\circ} \mathrm{C}$; Biokar Diagnostics). Then, Rapid'L.mono medium (Bio-Rad) was inoculated with 
$100 \mu \mathrm{L}$ of Fraser enrichment and incubated for $24 \mathrm{~h}$ at $37^{\circ} \mathrm{C}$. Typical positive colonies (blue colonies) were purified on tryptone soya agar medium with $5 \%$ sheep blood $\left(24 \mathrm{~h}\right.$ at $37^{\circ} \mathrm{C}$; Oxoid).

Presumptive L. monocytogenes isolates were confirmed by the rhamnose fermentation test (TCI AMERICA) in Remel Purple Broth Base medium (Thermo Fisher Scientific) as well as by multiplex PCR. For each suspected isolate, DNA was extracted with $50 \mu \mathrm{L}$ of 10\% Chelex 100 Resin solution (Bio-Rad Laboratories). Samples were heated $\left(30 \mathrm{~min}\right.$ at $55^{\circ} \mathrm{C}$ followed by 15 min at $98^{\circ} \mathrm{C}$ ) and centrifuged at $4^{\circ} \mathrm{C}$ for 5 min at 10,000 $\times g$ (Eppendorf Micro Centrifuge $5415 \mathrm{C}$, Brinkmann Instrument Inc.). The prs (370 bp) and prfA (274 bp) genes were amplified using primers LIP1, LIP2, prsF, and prsR (Kérouanton et al., 2010). The reaction for one volume of $50 \mu \mathrm{L}$ contained $1.0 \times$ reaction buffer, $2.0 \mathrm{mM} \mathrm{MgCl}$, $0.2 \mathrm{mM}$ dNTPs mix, primers (Supplemental Table S2; https://github.com/CRSV), 1.0 U of polymerase, and $2 \mu \mathrm{L}$ of DNA $(10 \mathrm{ng} / \mu \mathrm{L})$. We performed PCR using an Eppendorf Mastercycler nexus (Eppendorf Canada) using the following program: lid temperature $105^{\circ} \mathrm{C}$, the first denaturation for step 3 min at $94^{\circ} \mathrm{C}$ followed by $35 \mathrm{PCR}$ cycles consisting of denaturation $\left(94^{\circ} \mathrm{C}\right.$ for $\left.40 \mathrm{~s}\right)$, annealing $\left(53^{\circ} \mathrm{C}\right.$ for $\left.45 \mathrm{~s}\right)$, and extension $\left(72^{\circ} \mathrm{C}\right.$ for $1 \mathrm{~min}$ and $\left.15 \mathrm{~s}\right)$. The final step was set at $72^{\circ} \mathrm{C}$ for $7 \mathrm{~min}$ and held at $4^{\circ} \mathrm{C}$ (Kérouanton et al., 2010). Positive (L. monocytogenes ATCC 63253) and negative (Listeria ivanovii ATCC 19119 and water) controls were used. Electrophoresis on $2 \%$ agarose gel was performed with PCR products as follows: $5 \mu \mathrm{L}$ of DNA products was mixed with $1 \mu \mathrm{L}$ of loading buffers. To assess PCR amplicon size, the Invitrogen 1-kb ladder (Thermo Fisher Scientific) was used. Gel migration was performed at $120 \mathrm{~V}$ for $35 \mathrm{~min}$. Bands were visualized under UV lamps with the use of SYBR Safe DNA gel stain (Thermo Fisher Scientific).

Bacterial Counts of Mastitis-Related Bacteria. A total of $10 \mathrm{~g}$ of bedding sample was added to $90 \mathrm{~mL}$ of tryptone-salt solution (1\% tryptone and $8.5 \%$ sodium chloride; Thermo Fisher Scientific). Series of dilutions $\left(10^{0}\right.$ to $\left.10^{-4}\right)$ were performed in tryptone-salt for each sample. Based on preliminary enumeration tests, dilutions $10^{0}$ and $10^{-2}$ for unused straw and dilutions $10^{-2}$ and $10^{-4}$ for unused and used RMS samples, as well as used straw, were kept for enumeration with an automatic spiral plater (Interscience). Each sample dilution was plated on Vogel and Johnson agar medium (BD Difco) with 1\% tellurite solution (Sigma-Aldrich), MacConkey Agar medium (BD Difco), Edwards (modified) medium (Oxoid Canada) with 5\% sheep defibrinated blood (Oxoid Canada), and tryptone soy agar medium with 5\% sheep blood (Oxoid). Those mediums were used for enumeration of Staphylococcus spp. (Vogel and Johnson agar), coliforms (MacConkey), Streptococcus spp. (Edwards), and total mesophilic bacteria (tryptone soy agar with blood). Plates were incubated for $24 \mathrm{~h}$ at $35 \pm 2^{\circ} \mathrm{C}$. Enumerations of colony forming units were performed as recommended, using a count grid. The accuracy of the morphologicalbased identification for 5 Staphylococcus spp. and 5 Streptococcus spp. isolates was confirmed with API ID strips (bioMérieux). The morphologically based identification was correct for $100 \%$ of isolates tested. Escherichia coli and Klebsiella spp. were enumerated separately based on the morphological appearance of the colonies (pink-to-red colonies on MacConkey were presumed to be Klebsiella spp.). To validate the accuracy of the morphological-based identification for separating Klebsiella from $E$. coli colonies, we further confirmed identification of $20 \mathrm{E}$. coli and 20 Klebsiella spp. isolates using matrix-assisted laser desorption/ ionization time-of-flight (MALDI-TOF) mass spectrometry (Bruker Ltd.). The morphologically based identification was correct for $100 \%$ of isolates tested. All enumeration results were transformed in $\log 10$ $\mathrm{cfu} / \mathrm{g}$ before statistical analyses and, for each sample and each medium, the mean of the 2 enumerations was computed and used in subsequent statistical analyses.

\section{Statistical Analyses}

Sequencing Analyses. Microbiota analysis was performed in RStudio (v. 1.2.5033) using $\mathrm{R}$ version 3.6.1 (https://www.r-project.org/). We used ggplot2, vegan, dplyr, scales, grid, reshape2, phyloseq, ape, gplots, lme4, phangorn, plotly, tidyr, VennDiagram, data.table, and RColorBrewer packages. A MannWhitney statistical test was performed to compare the mean relative abundances of the main phyla presented in the stack bar charts $(P<0.05)$. The numbers of OTU observed, inverted Simpson's index, and Shannon evenness were calculated. The results were compared between the previously mentioned groups using Kruskal-Wallis and Wilcoxon tests $(P<0.05)$. Beta diversity was analyzed by first looking at the microbiota structure using a distance matrix, comparing all samples and groups with the Jaccard and Bray-Curtis dissimilarity indices. The results were visualized using a nonmetric multidimensional scaling (NMDS) plot. Bedding types were compared using the ADONIS test. The Venn diagram was constructed with a cut-off of $90 \%$ for OTU present in the bedding samples; in other words, an OTU was conserved if it was present in $90 \%$ of bedding samples, regardless of the category. This cut-off was used because a large number of OTU were 
found in bedding samples, and we wanted to avoid OTU that were found in only few samples.

\section{Analyses of Data Related to Bacterial Counts and Presence of Zoonotic Pathogens}

The probabilities of recovering Salmonella spp. or L. monocytogenes in unused and used bedding were compared between RMS and straw-bedded herds. To achieve this, Fisher exact tests were used.

Counts of Staphylococcus spp., Streptococcus spp., Klebsiella spp., E. coli, and total mesophilic flora were compared between unused and used RMS, between unused and used straw, between unused RMS and unused straw, and, finally, between used RMS and straw. To achieve this, a linear regression model was developed for each bacterial group, with $\log 10 \mathrm{cfu} / \mathrm{g}$ of the bacterial group as the outcome, and fixed predictors as follows: type of bedding (RMS vs. straw), the status of the bedding (unused vs. used), and the interaction term between type of bedding and bedding status. Moreover, housing type (freestall vs. tiestall) was included in the model as a fixed predictor because it was hypothesized to act as a confounder of the relationship between bedding type and bacterial counts when comparing RMS to straw. To evaluate whether correlation between the different predictors (especially between type of bedding and housing type) would induce collinearity problems, the variance inflation factor (VIF) was computed. The VIF represents the effect of entering a new variable in a model on the variance of the coefficients of the other variables (Dohoo et al., 2009). According to Dohoo et al. (2009), a VIF $>10.0$ is considered to be indicative of serious collinearity. When comparing the different groups, a Tukey-Kramer adjustment was used to take into account the multiple comparisons made. Assumptions of normality and homoscedasticity of the residuals were verified using visual inspection of a histogram of the residuals and of a scatter plot of the residuals against the predicted $\log _{10} \mathrm{cfu} / \mathrm{g}$ values, respectively.

We then compared bacterial counts in unused RMS as a function of the method used to produce the RMS for the most commonly used methods (separation followed by maturation in an opened or enclosed container and separation followed by maturation in a pile). Again, a linear regression model was applied, with RMS unused bedding data alone and with count (in $\log _{10} \mathrm{cfu} / \mathrm{g}$ ) of a group of bacteria as outcome, and type of method used to produce the RMS as fixed predictor. A data set containing all of the variables needed to reproduce the statistical analyses conducted using classical microbiological data is publicly available (Supplemental Data S1, https://doi.org/10.5683/SP2/8HRWGR; Dufour, 2021).

\section{RESULTS}

\section{Farm Recruitment and RMS Production}

We recruited 27 RMS farms and 61 straw-bedded farms for the study $(\mathrm{n}=88$; Table 1$)$. Seventeen farms used a screw-press system for removing the liquid fraction from the solid fraction, and 10 farms used a roller press system. One farm used the RMS immediately after the separation step without further maturation. After liquid extraction, 26 farms let the RMS solid fraction mature before use as follows: in an open heap (10 farms), in an opened or enclosed container (usually closed on all sides with only the upper part being exposed to air; 13 farms), or a rotating drum (2 farms; Table 1). One farm used an anaerobic digester. The maturation time varied from $9 \mathrm{~h}$ to $9 \mathrm{~d}$, except for the farm using anaerobic digestion, which was completed over $30 \mathrm{~d}$. The RMS was added to the stalls 0.14 to 4 times per day $(24 \mathrm{~h})$. The straw-bedded farms added the bedding 1 to 4 times per day (24 h; Table 1 ).

\section{Microbiota Description}

A total of 10,044,820 sequences were retained after bioinformatics treatment, with Mothur yielding a total of 117,545 unique sequences. Because we set the clustering methods to "unique," each unique sequence was assigned to its own OTU. Samples were normalized to 18,950 sequences (size of the smallest sample). The maximum, median, and mean numbers of sequences per sample were $69,876,54,060$, and 53,785, respectively. The sequences and OTU numbers of each control are presented in Table 2. Venn diagrams were constructed to present the genera that were unique and shared between bedding groups. To achieve this, the sequences were merged at the genus taxonomic rank, yielding a total of 3,712 different genera. Genera absent in at least $90 \%$ of bedding types and bedding status were discarded. Each component (type and status) was calculated separately before comparison. Therefore, 748 genera constituted the total bedding microbiota, and the bedding core microbiota (shared by all bedding: unused RMS, used RMS, unused straw, and used straw) was 303.

Unused Bedding Microbiota. Microbiota from unused RMS samples are compared with those of unused straw samples in Figure 2. The major phyla $(\mathrm{n}=27)$ were represented in both unused RMS and straw samples, but in different proportions. Effectively, most of the major phyla were present in various proportions (except for Actinobacteria phylum; $P=0.25$ ), confirming a clear difference in phylum composition of the respective microbiota between unused RMS 
and straw bedding. Unused RMS and straw samples were composed as follows: Proteobacteria (RMS 38\%, straw 69\%; $P<0.001$ ), Firmicutes (RMS 32\%, straw $2 \% ; P<0.001)$, Bacteroidetes (RMS 14\%, straw 21\%; $P=0.007$ ), and Actinobacteria (RMS 7\%, straw 6\%; $P=0.25$; Figure 2). Also, we observed DeinococcusThermus (1\%; 95\% CI: 0.01-16\%) in unused RMS, but not in unused straw (Figure 2A). At the genus level, we obtained a total of 861 genera in unused RMS and 870 in unused straw. Genera with a relative abundance of greater than $10 \%$ are showed in Figure 3. Among them, only Pseudomonas genus was present in both RMS and straw.

The $\alpha$-diversity analyses showed differences between unused RMS and unused straw for all measured diversity indices (observed: $P<0.001$; Shannon: $P<$ 0.001; InvSimpson: $P=0.017$; Figure 4A). In general, diversity was more important in unused RMS than in unused straw bedding. The $\beta$-diversity was also investigated and illustrated by NMDS (Figure 4B), and the microbiota structures compared using Jaccard and Bray-Curtis indices and the ADONIS test. Bedding type (unused RMS vs. unused straw) was associated with sample microbiota structure $(P<0.001)$.

Furthermore, a table illustrating genera unique to a bedding type was constructed using Venn diagrams. A total of 217 unique genera in unused RMS compared with 103 unique genera in straw were observed (Supplemental Table S3; https://github.com/CRSV). A higher presence of unique genera in unused RMS samples appeared compared with unused straw samples, but 312 genera were shared between the unused RMS and unused straw bedding samples (Supplemental Table S3).

We detected L. monocytogenes more frequently in unused RMS (30\%; 95\% CI: 15-50\%) compared with in unused straw (11\%; 95\% CI: 5-23\%). Moreover, we detected Salmonella spp. (K serogroup) in 11\% (95\% CI: 3-30\%) of unused RMS, but in only 1 (1.6\%; $95 \%$ CI: $1-8 \%$ ) of the unused straw samples.

We observed significantly higher bacterial counts, in general, in unused RMS compared with unused

Table 2. Sequences and operational taxonomic unit (OTU) numbers in positive and negative controls ${ }^{1}$

\begin{tabular}{lrr}
\hline Control & $\begin{array}{c}\text { Sequence } \\
\text { number }\end{array}$ & $\begin{array}{c}\text { OTU } \\
\text { number }\end{array}$ \\
\hline PCR negative & 184 & 35 \\
DNA extraction negative 1 & 9,658 & 850 \\
DNA extraction negative 2 & 88 & 15 \\
PCR zymo positive & 54,456 & 286 \\
DNA extraction zymo positive 1 & 53,587 & 168 \\
DNA extraction zymo positive 2 & 47,936 & 212 \\
\hline
\end{tabular}

${ }^{1}$ Zymo positive $=$ DNA extracted from zymo microbial community; negative $=$ PCR water used instead of DNA sample. straw, except for Klebsiella spp. (Table 3). Total mesophilic flora counts were estimated to be $0.9 \log 10$ $\mathrm{cfu} / \mathrm{g}$ higher in unused RMS bedding than in unused straw. Staphylococcus spp., Streptococcus spp., and E. coli counts were estimated to be 2.6, 1.6, and $2.0 \mathrm{log}$ $10 \mathrm{cfu} / \mathrm{g}$ higher, respectively, in unused RMS bedding (Table 4). On the other hand, Klebsiella spp. counts were significantly lower in unused RMS samples by an average of $1.6 \log 10 \mathrm{cfu} / \mathrm{g}$ (Table 4).

Used Bedding Microbiota. Microbiota of the samples originating from used RMS samples were compared with those of used straw samples (Figure 5). We observed 32 phyla in used RMS and 29 in used straw samples. Most phyla were observed in similar proportions in both used RMS and used straw samples. Used RMS and straw microbiota were composed mostly of Proteobacteria (RMS 53\%, straw 46\%; $P=0.009$ ), Bacteroidetes (RMS 25\%, straw 22\%; $P=0.26$ ), Firmicutes (RMS 10\%, straw 20\%; $P<0.001$ ), and Actinobacteria (RMS 8\%, straw 10\%; $P=0.036$; Figure 6). The RMS was also composed of Deinococcus-Thermus (1\%;95\% CI: 0.0001-22\%; Figure 5A).

Totals of 927 and 1,054 genera were found in used RMS and used straw samples, respectively. Genera with a relative abundance of greater than $10 \%$ are represented in Figure 6. Briefly, Acinetobacter, Flavobacteriaceae unclassified, Pseudomonas, and Psychrobacter genera were found both in used RMS and used straw bedding. Furthermore, 113 unique genera were observed in used RMS compared with 136 in used straw; 390 genera were shared between used RMS and used straw (Supplemental Table S4; https://github.com/CRSV).

The $\alpha$-diversity was investigated, and no differences could be observed in the different measures of diversity between used RMS and used straw (observed: $P$ $=0.068$; Shannon: $P=0.81$; InvSimpson: $P=0.70$; Figure $4 \mathrm{~A}$ ). In used bedding samples, the microbiota structure was associated with bedding type, similar to that observed in unused beddings $(P<0.001$; Figure 4B).

We detected L. monocytogenes in $48 \%$ (95\% CI: 29 $67 \%)$ of used RMS samples compared with $20 \%$ (95\% CI: $11-32 \%$ ) of used straw samples. Salmonella spp. (K, $\mathrm{C} 1$, and $\mathrm{B}$ serogroups) were detected significantly more frequently in used RMS (15\%; 95\% CI: 5-35\%) than in used straw samples (2\%; $95 \%$ CI: $0-10 \%)$.

Regarding counts of udder health-related bacteria, a higher total mesophilic bacterial count was recorded in used RMS (9.5 log $10 \mathrm{cfu} / \mathrm{g} ; 95 \%$ CI: 9.2-9.8) compared with used straw bedding (8.6 log $10 \mathrm{cfu} / \mathrm{g} ; 95 \%$ CI..4-8.9) as well as higher E. coli counts (RMS: $5.4 \mathrm{log}$ $10 \mathrm{cfu} / \mathrm{g} ; 95 \%$ CI: 5.1-5.7; straw: $4.6 \log 10 \mathrm{cfu} / \mathrm{g} ; 95 \%$ CI: 4.3-4.9; Table 3). On the other hand, used straw samples had significantly higher Streptococcus spp. 


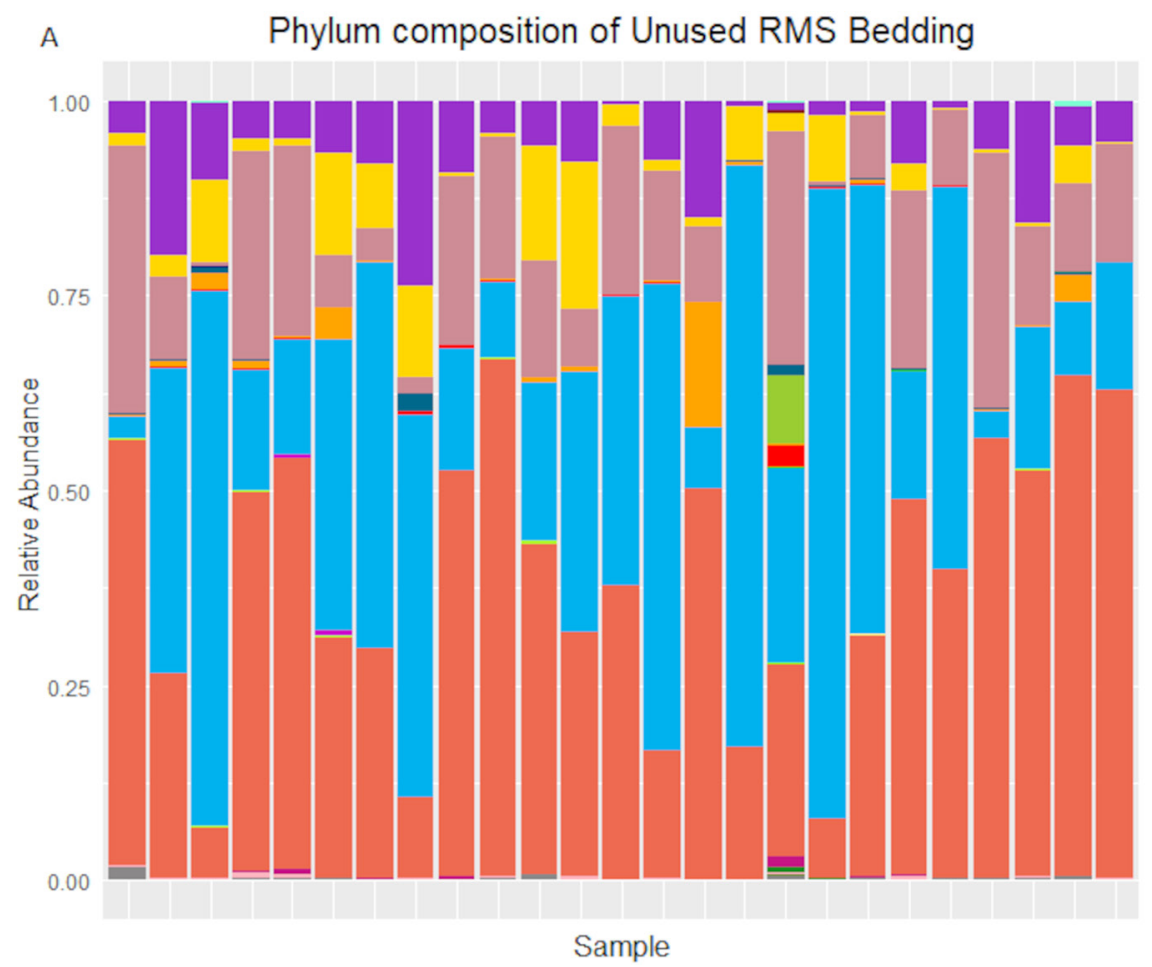

\begin{tabular}{|c|c|}
\hline \multicolumn{2}{|l|}{ Phylum } \\
\hline Acidobacteria & Firmicutes \\
\hline Actinobacteria & Fusobacteria \\
\hline Archaea_unclassified & Gemmatimonadete \\
\hline Armatimonadetes & Hydrogenedentes \\
\hline Bacteria_unclassified & Lentisphaerae \\
\hline Bacteroidetes & Pacearchaeota \\
\hline BRC1 & Parcubacteria \\
\hline candidate_division_WPS-1 & Planctomycetes \\
\hline Candidatus_Saccharibacteria & Proteobacteria \\
\hline Chlamydiae ${ }^{-}$ & Spirochaetes \\
\hline Chloroflexi & SR1 \\
\hline Cloacimonetes & Synergistetes \\
\hline Deinococcus-Thermus & Tenericutes \\
\hline Elusimicrobia & Thaumarchaeota \\
\hline Euryarchaeota & Thermotogae \\
\hline Fibrobacteres & Verrucomicrobia \\
\hline
\end{tabular}

B

Phylum composition of Unused Straw Bedding

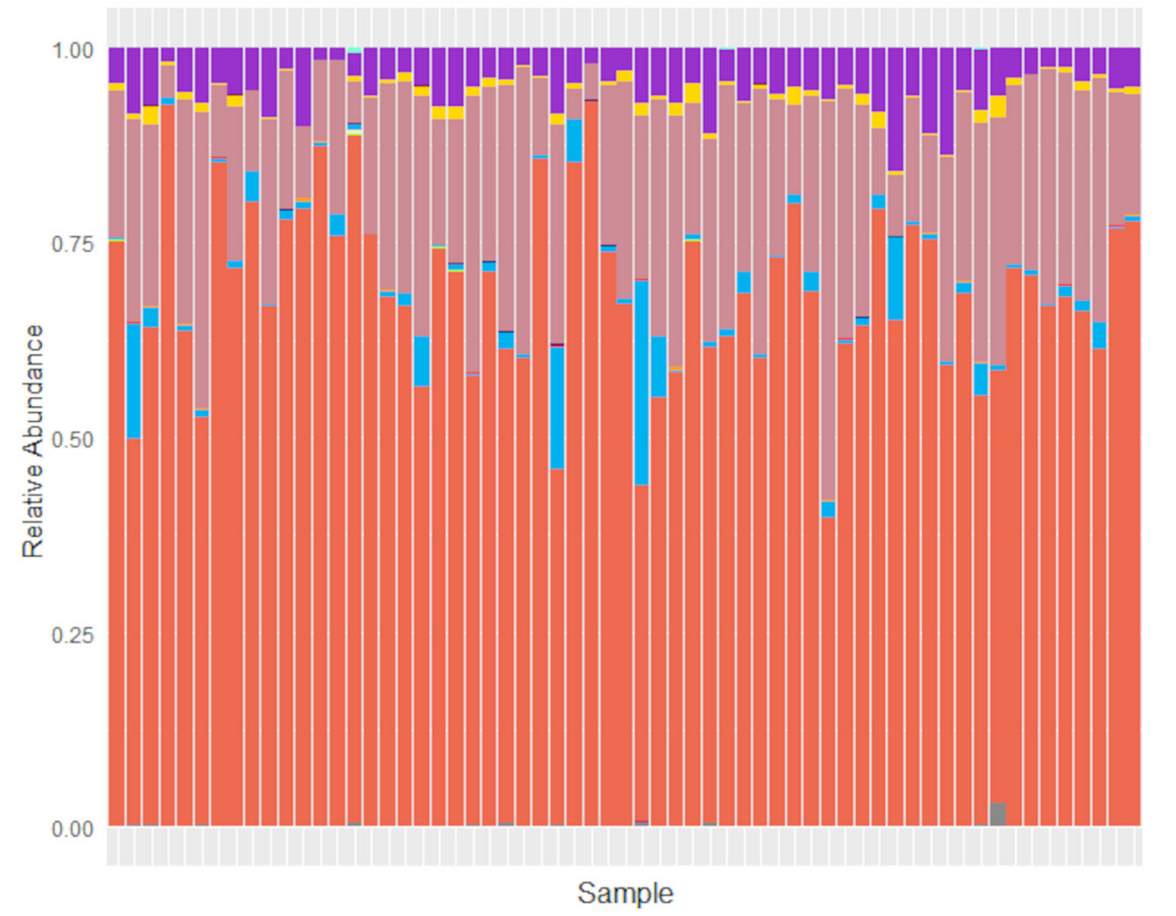

\begin{tabular}{ll}
\multicolumn{1}{c}{ Phylum } & \\
Acidobacteria & Fusobacteria \\
Actinobacteria & Gemmatimonadetes \\
Armatimonadetes & Latescibacteria \\
Bacteria_unclassified & Lentisphaerae \\
Bacteroidetes & Nitrospirae \\
BRC1 & Parcubacteria \\
candidate_division_WPS-1 & Planctomycetes \\
Candidatus_Saccharibacteria & Proteobacteria \\
Chlamydiae & Spirochaetes \\
Chloroflexi & SR1 \\
Cloacimonetes & Synergistetes \\
Deinococcus-Thermus & Tenericutes \\
Elusimicrobia & Thaumarchaeota \\
Euryarchaeota & Verrucomicrobia \\
Fibrobacteres & Woesearchaeota \\
Firmicutes &
\end{tabular}

Figure 2. Phyla repartition in unused recycled manure solids (RMS; A; $n=27)$ and unused straw $(\mathrm{B} ; \mathrm{n}=61)$ bedding samples. Abundance filter was set to zero, and 32 and 31 phyla were obtained for unused RMS and unused straw, respectively. 
A Genus Composition of Unused RMS Bedding

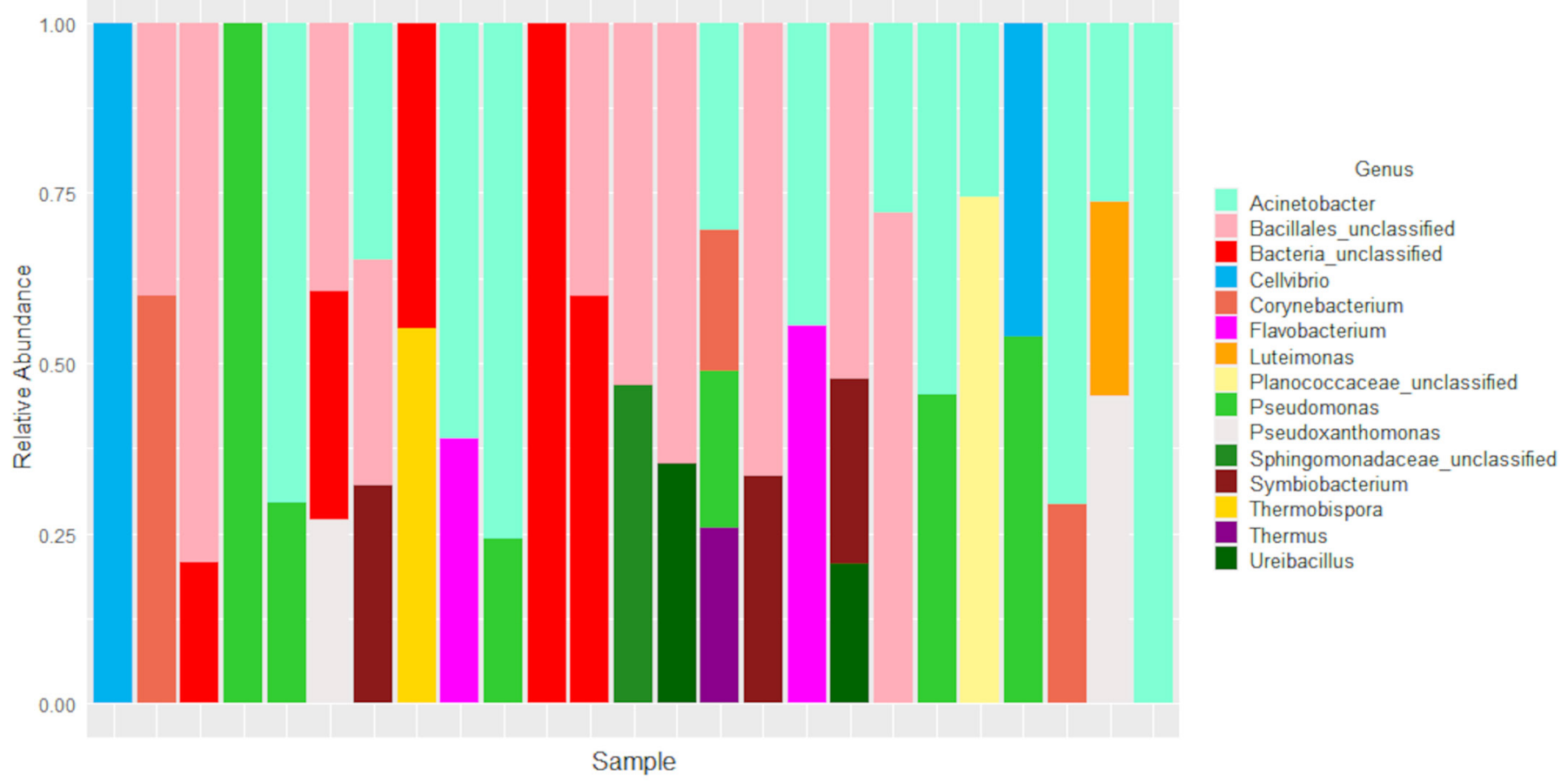

B Genus Composition in Unused Straw Bedding

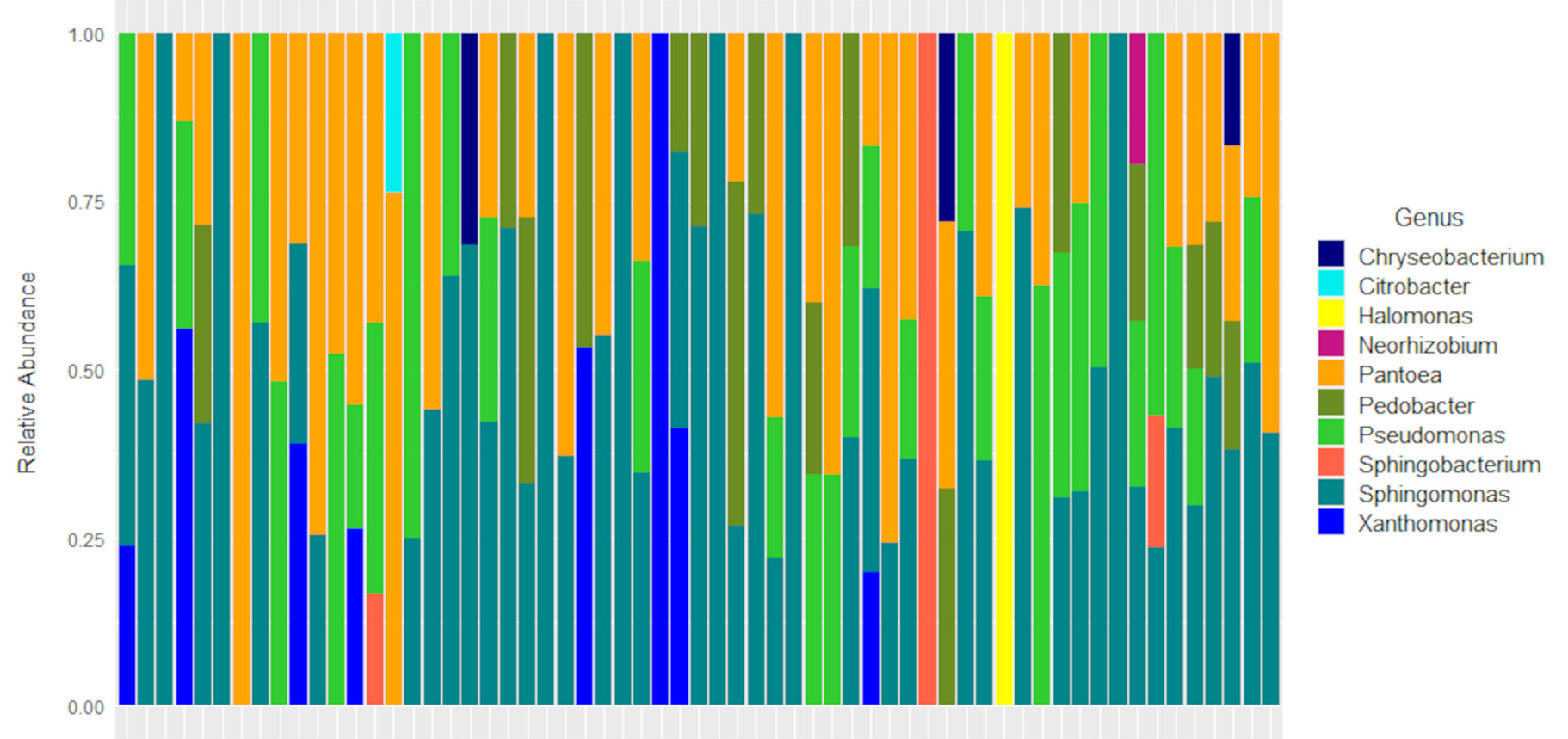

Sample

Figure 3. Genera repartition of unused recycled manure solids (RMS; A; $n=27)$ and unused straw $(B ; n=61)$ bedding samples. Abundance filter was set to 0.10 , and 15 genera were obtained for unused RMS and 10 genera for unused straw. 
A

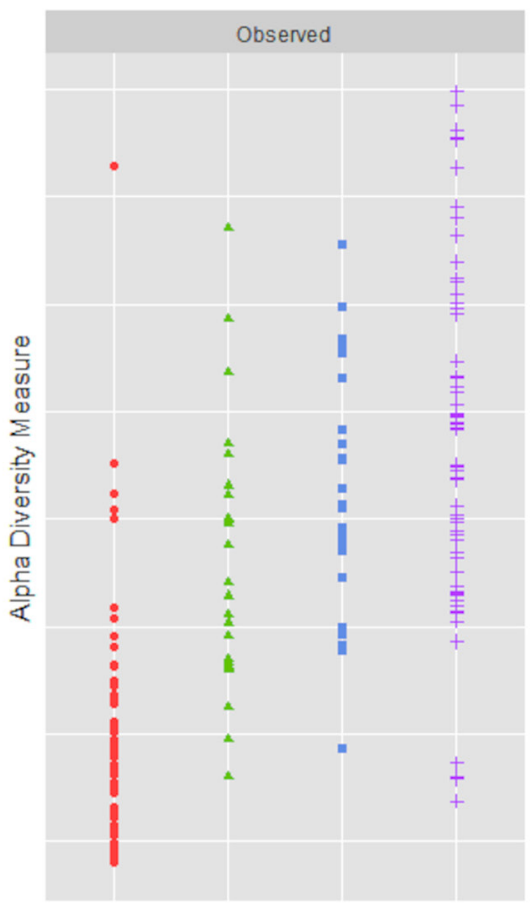

\section{Alpha diversity of bedding}

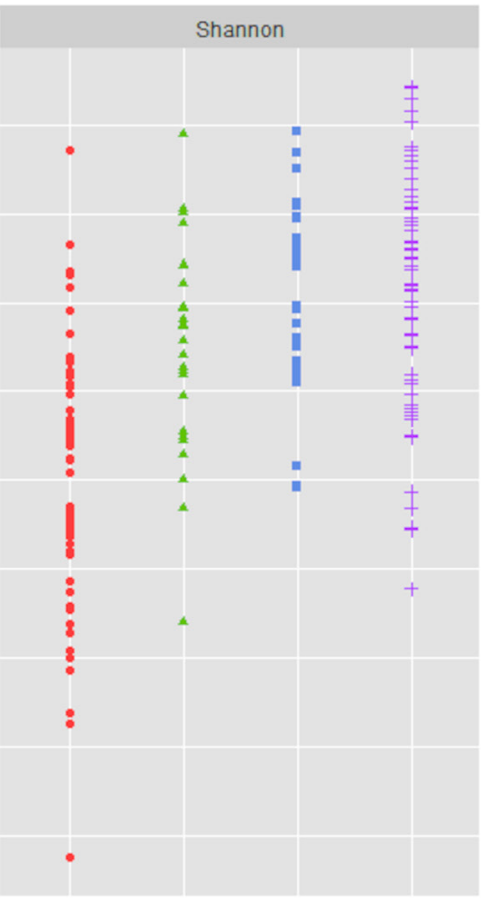

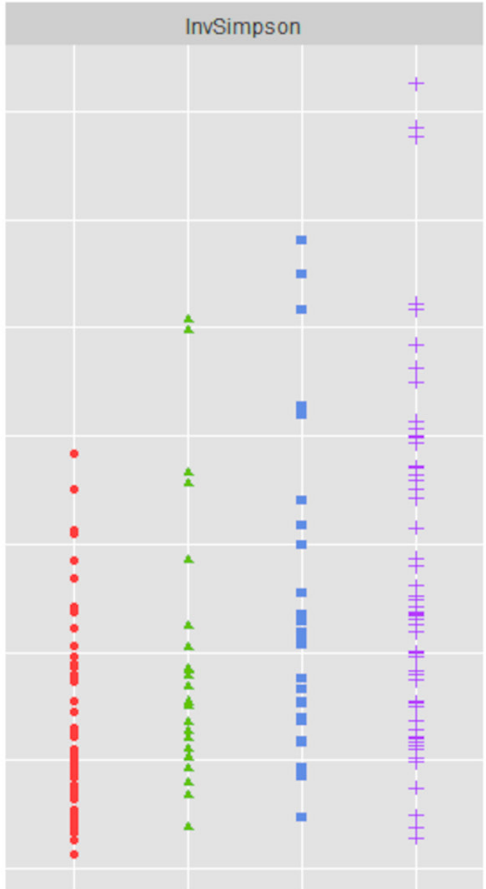

\section{Bedding_status}

- Straw_Unused

4 RMS_Unused

- RMS_Used

+ Straw_Used

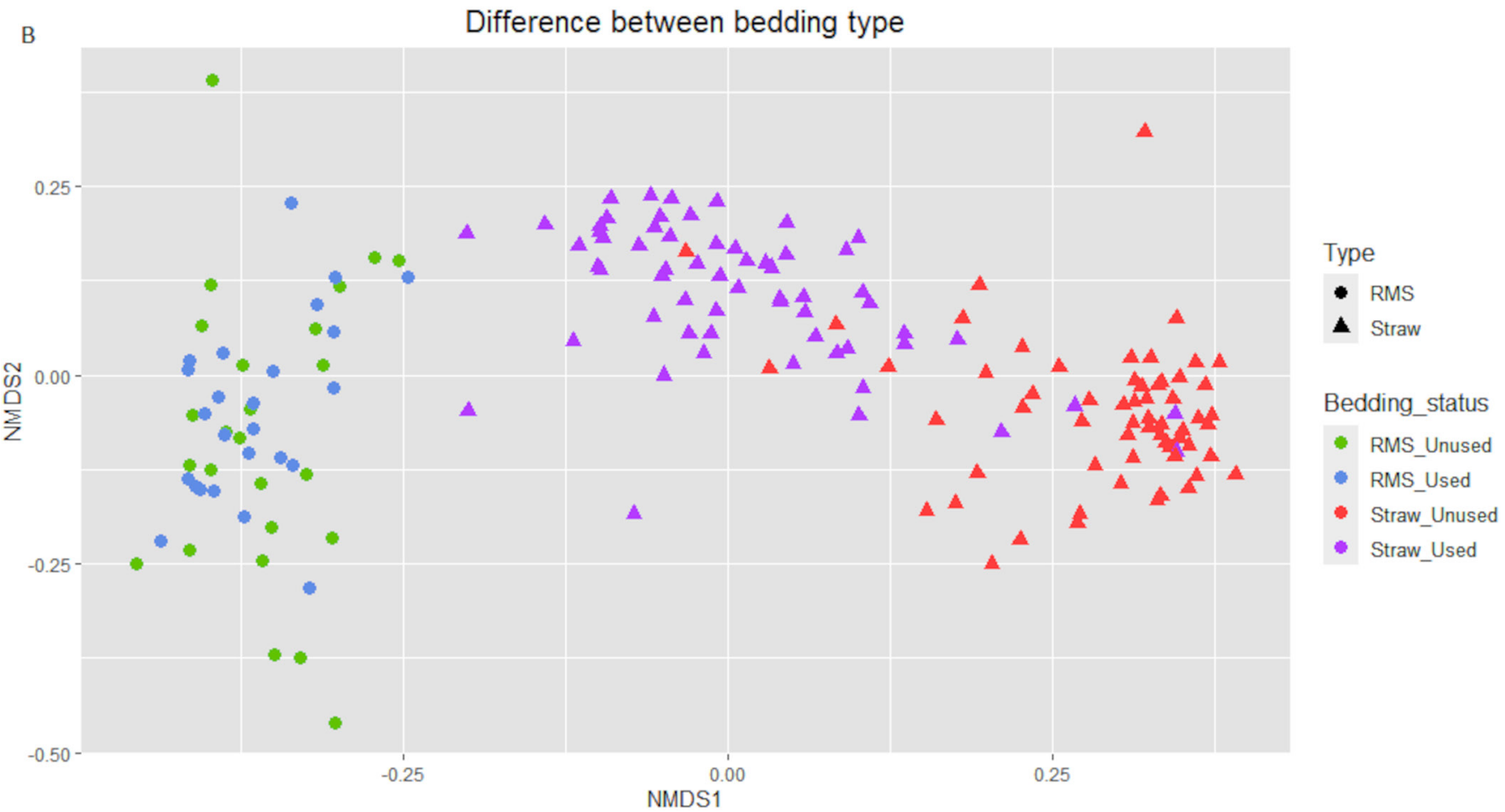

Figure 4. Microbiota analysis on bedding samples. (A) Observed, Shannon, and Inverse Simpson index measures of diversity in used and unused recycled manure solids (RMS) and straw beddings. (B) Nonmetric multidimensional scaling (NMDS) plot illustrating microbiota $\beta$-diversity according to bedding type (RMS or straw) and status (used or unused). Each point represents a single bedding sample microbiota based on 107,934 operational taxonomic units. Jaccard and Bray-Curtis indices and ADONIS tests $(P<0.001)$ were performed in RStudio (v. 1.2.5033) for statistical analysis. 
Table 3. Least squares means estimated bacterial counts for different groups of bacteria in unused and used recycled manure solids (RMS) and straw bedding

\begin{tabular}{|c|c|c|c|c|c|}
\hline \multirow[b]{2}{*}{ Bacterial group } & \multirow[b]{2}{*}{ Status } & \multicolumn{2}{|c|}{ RMS } & \multicolumn{2}{|c|}{ Straw } \\
\hline & & $\log \mathrm{cfu} / \mathrm{g}^{1}$ & $95 \% \mathrm{CI}$ & $\log \mathrm{cfu} / \mathrm{g}^{1}$ & $95 \% \mathrm{CI}$ \\
\hline \multirow{2}{*}{ Total mesophilic flora } & Unused & $8.0^{\mathrm{a}}$ & $7.7,8.3$ & $7.1^{\mathrm{b}}$ & $6.8,7.4$ \\
\hline & Used & $9.5^{\mathrm{c}}$ & $9.2,9.8$ & $8.6^{\mathrm{d}}$ & $8.4,8.9$ \\
\hline \multirow[t]{2}{*}{ Staphylococci } & Unused & $5.4^{\mathrm{a}}$ & $5.0,5.8$ & $2.8^{\mathrm{b}}$ & $2.4,3.1$ \\
\hline & Used & $7.0^{\mathrm{c}}$ & $6.6,7.4$ & $6.8^{\mathrm{c}}$ & $6.4,7.1$ \\
\hline \multirow{2}{*}{ Streptococci } & Unused & $5.0^{\mathrm{a}}$ & $4.5,5.5$ & $3.4^{\mathrm{b}}$ & $3.0,3.9$ \\
\hline & Used & $6.2^{\mathrm{c}}$ & $5.7,6.8$ & $7.4^{\mathrm{d}}$ & $6.9,7.8$ \\
\hline \multirow[t]{2}{*}{ Escherichia coli } & Unused & $4.4^{\mathrm{a}}$ & $4.1,4.7$ & $2.4^{\mathrm{b}}$ & $2.1,2.7$ \\
\hline & Used & $5.4^{\mathrm{c}}$ & $5.1,5.7$ & $4.6^{\mathrm{a}}$ & $4.3,4.9$ \\
\hline \multirow[t]{2}{*}{ Klebsiella spp. } & Unused & $3.7^{\mathrm{a}}$ & $3.3,4.1$ & $5.3^{\mathrm{b}}$ & $4.9,5.7$ \\
\hline & Used & $5.2^{\mathrm{b}, \mathrm{c}}$ & $4.8,5.6$ & $5.8^{\mathrm{c}}$ & $5.5,6.2$ \\
\hline
\end{tabular}

${ }^{\mathrm{a}-\mathrm{d}}$ For a given bacterial group, estimates within a row or column with different superscripts were considered to be different $(P<0.05)$ after adjusting for multiple comparison using the Tukey-Kramer adjustment and for confounding by housing type (freestall vs. tiestall).

${ }^{1}$ Least squares means estimate.

counts (7.4 log $10 \mathrm{cfu} / \mathrm{g} ; 95 \%$ CI: 6.9-7.8) than did used RMS samples (6.2 log 10 cfu/g; 95\% CI: 5.7-6.8; Table $3)$. No substantial differences between used RMS and used straw bedding for Staphylococcus spp. (RMS: 7.0 $\log 10 \mathrm{cfu} / \mathrm{g} ; 95 \%$ CI: 6.6-7.4; straw: $6.8 \log 10 \mathrm{cfu} / \mathrm{g}$; 95\% CI: 6.4-7.1) or for Klebsiella spp. counts (RMS: 5.2 $\log 10 \mathrm{cfu} / \mathrm{g} ; 95 \%$ CI: 4.8-5.6; straw: $5.8 \log 10 \mathrm{cfu} / \mathrm{g}$; 95\% CI: 5.5-6.2; Table 3) were recorded.

Evolution of Straw Microbiota with Use. Microbiota in the samples originating from unused straw were compared with those of used straw to describe the evolution of microbiota during use. We observed 31 phyla in unused straw and 29 phyla in used straw samples. Twenty-eight phyla were shared by unused and used straw samples. Relative abundance of Pro-

Table 4. Least squares means estimated bacterial counts (in log cfu/g) for different production groups of bacteria in unused recycled manure solids (RMS) bedding according to the method used for production

\begin{tabular}{lllc}
\hline & & \multicolumn{2}{c}{$\begin{array}{c}\text { RMS production } \\
\text { method }^{1}\end{array}$} \\
\cline { 3 - 4 } Bacterial group & Parameter & $\begin{array}{c}\text { S-MH } \\
(\mathrm{n}=10)\end{array}$ & $\begin{array}{c}\text { S-MB } \\
(\mathrm{n}=13)\end{array}$ \\
\hline Total mesophilic flora & LSM & 7.7 & 8.5 \\
& $95 \%$ CI & $7.1,8.3$ & $7.9,9.0$ \\
Staphylococci & LSM & $4.8^{\mathrm{a}}$ & $5.8^{\mathrm{b}}$ \\
Streptococci & $95 \%$ CI & $4.1,5.6$ & $5.2,6.5$ \\
Escherichia coli & LSM & $4.3^{\mathrm{a}}$ & $5.7^{\mathrm{b}}$ \\
Klebsiella spp. & $95 \%$ CI & $3.5,5.0$ & $5.1,6.4$ \\
& LSM & $4.2^{\mathrm{a}}$ & $4.9^{\mathrm{b}}$ \\
& $95 \%$ CI & $3.8,4.6$ & $4.6,5.3$ \\
\hline
\end{tabular}

${ }^{\mathrm{a}, \mathrm{b}}$ For a given bacterial group, estimates within a row with different superscripts were considered to be different $(P<0.05)$.

${ }^{1} \mathrm{~S}-\mathrm{MH}=$ separation followed with maturation in a heap; $\mathrm{S}-\mathrm{MB}=$ separation with maturation in an opened or enclosed container. teobacteria was greater in unused straw $(69 \%$ in unused vs. $46 \%$ in used straw bedding; $P<0.001)$, but Actinobacteria and Firmicutes were associated more with used straw (20\% and 10\%, respectively; $P<0.001 ; P$ $<0.001)$ compared with unused straw $(6 \%$ and $2 \%$, respectively). At the genus level, totals of 870 genera in unused straw samples and 1,054 genera in used straw samples were obtained. Among genera with a relative abundance of more than $10 \%, 6$ that were common to unused and used straw samples were noticed as follows: Chryseobacterium, Pantoea, Pedobacter, Pseudomonas, Sphingomonas, and Xanthomonas. Using the Venn diagrams, 390 genera common to unused and used straw, 25 genera unique to unused straw, and 209 genera unique to used straw (Supplemental Table S5; https:/ /github.com/CRSV) were observed. A higher presence of unique genera was observed in used straw than in unused straw samples.

A greater $\alpha$-diversity in used straw bedding samples compared with unused straw samples was revealed (observed: $P<0.001$; Shannon: $P<0.001$; InvSimpson: $P$ $<0.001$; Figure 4A). Regarding $\beta$-diversity, significant differences appeared in microbiota structure between unused and used straw $(P<0.001$; Figure 4B). Finally, the used straw samples had significantly higher bacterial counts than did unused straw samples for all bacterial groups, with differences ranging between 0.5 $\log 10 \mathrm{cfu} / \mathrm{g}$ (for Klebsiella spp.) to $4.0 \log 10 \mathrm{cfu} / \mathrm{g}$ (for Staphylococcus spp. and Streptococcus spp.; Table 3).

Evolution of RMS Microbiota with Use. Microbiota in the samples originating from the unused RMS samples were compared with those from used RMS samples. Thirty phyla were common to unused and used RMS samples. The relative abundance of Firmicutes was significantly greater in unused RMS compared with used RMS samples. On the other hand, the 


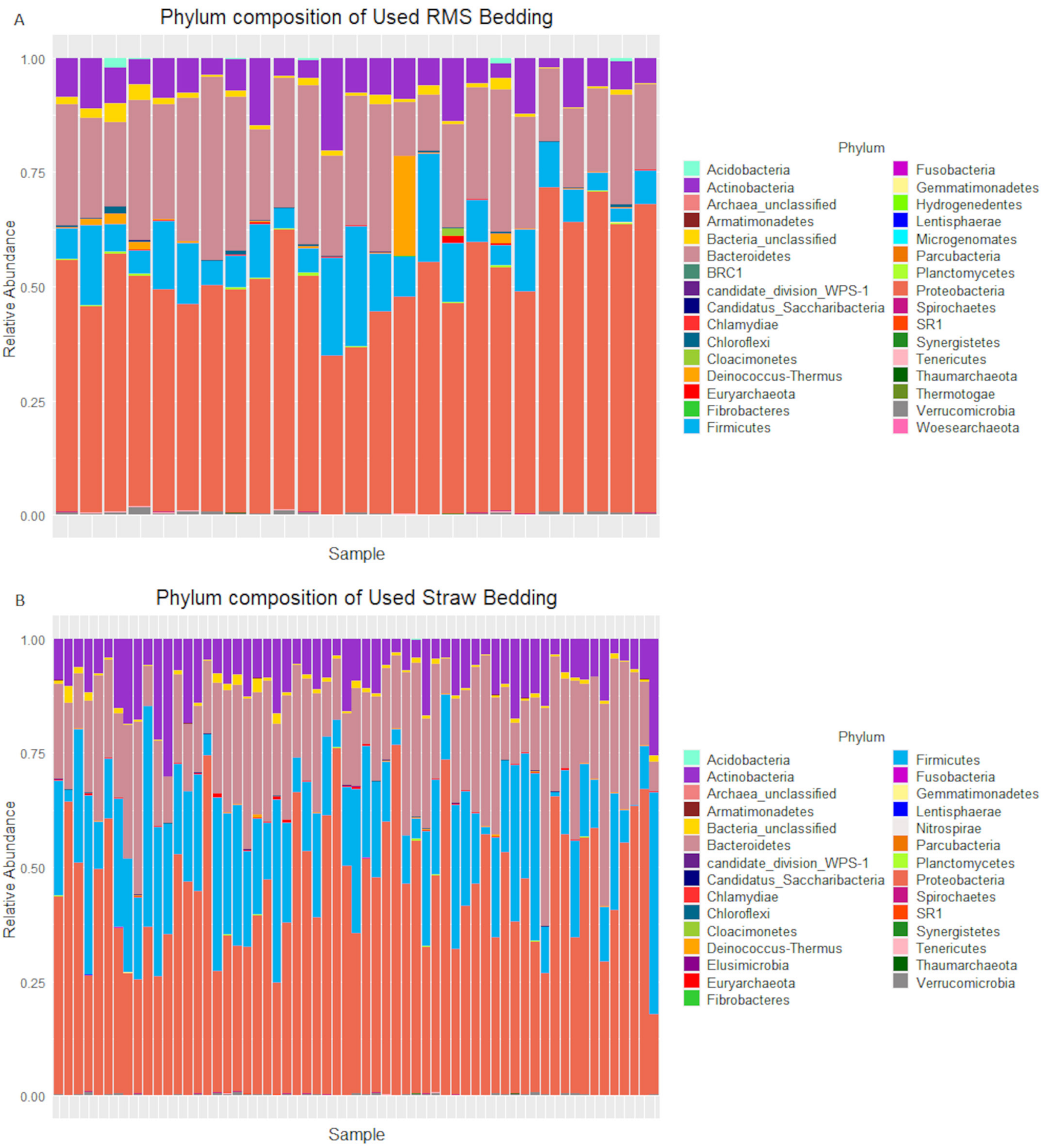

Figure 5. Phyla repartition in used recycled manure solids (RMS; A; $\mathrm{n}=27)$ and used straw $(\mathrm{B} ; \mathrm{n}=61)$ bedding samples. Abundance filter was set to zero, and 32 and 29 phyla were obtained for used RMS and used straw bedding, respectively. 


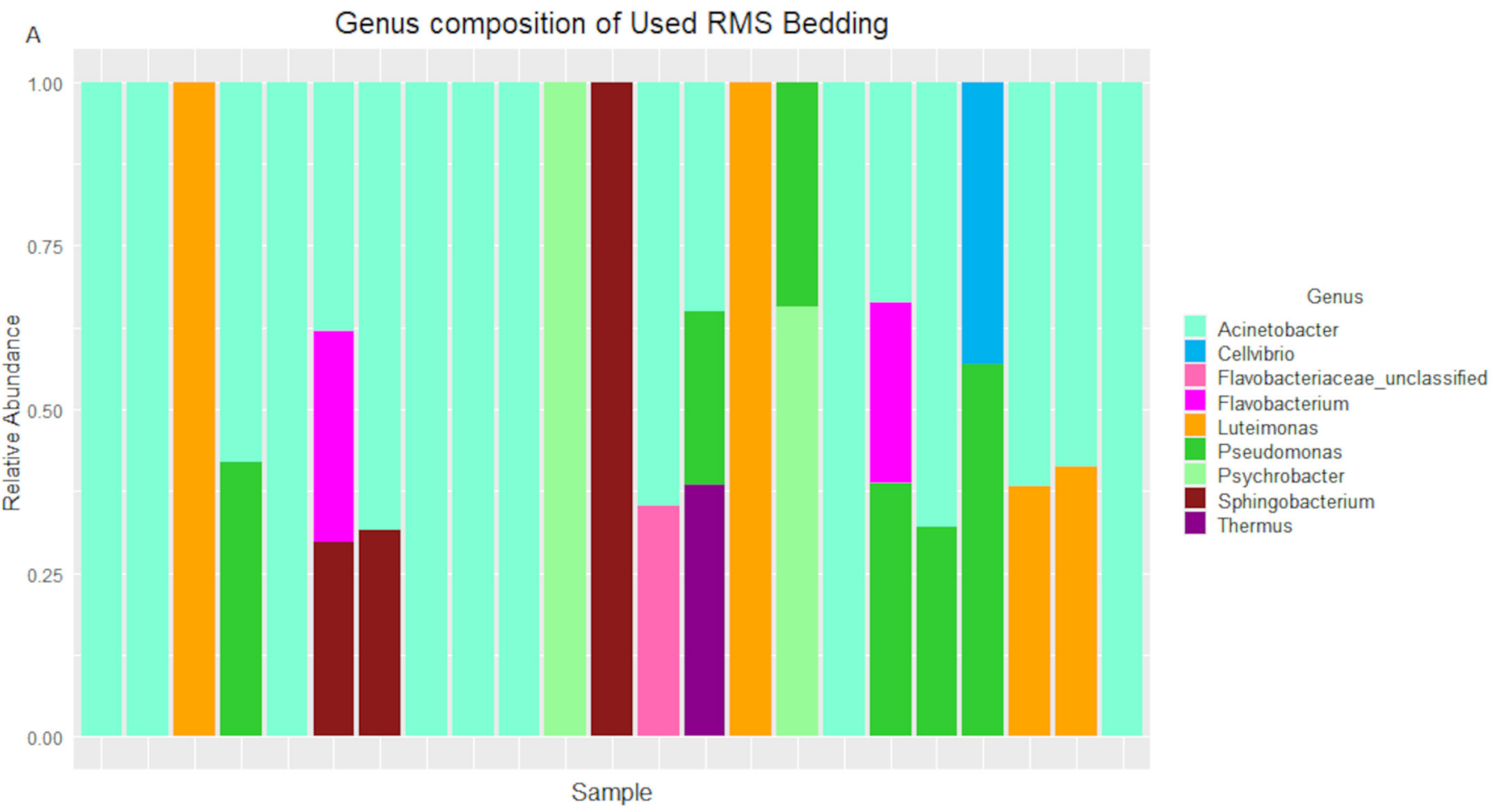

B Genus composition of Used straw Bedding

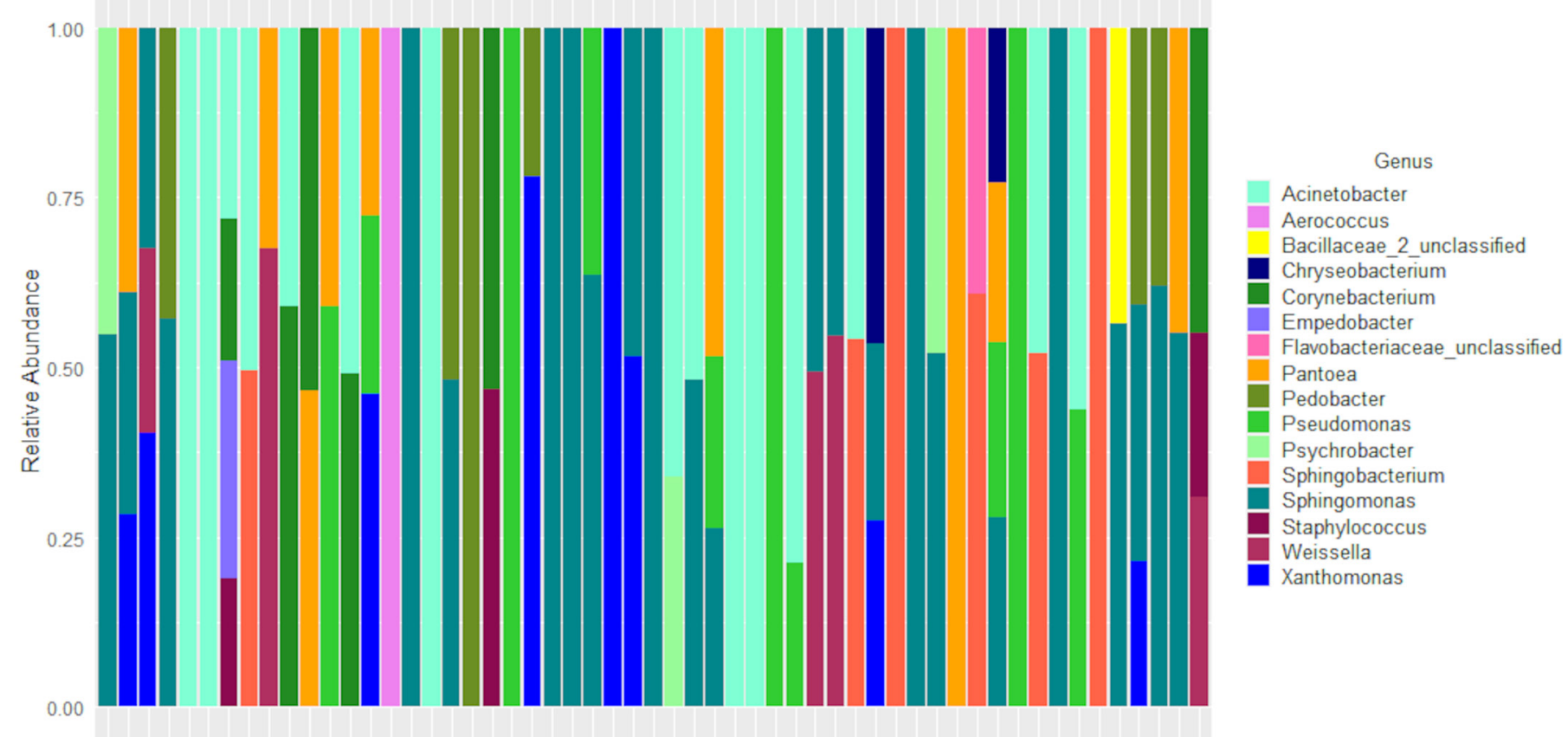

Sample

Figure 6. Genera repartition in used recycled manure solids (RMS; A; $n=27)$ and unused straw $(B ; n=61)$ bedding samples. Abundance filter was set to 0.10 , and 9 genera were obtained for used RMS and 16 genera for used straw. 
relative abundances of Proteobacteria and Bacteroidetes were greater in used than in unused RMS samples $(P$ $=0.001 ; P<0.001)$. At the genus level, used RMS contained more genera (927) compared with unused RMS (861) samples. Among genera with a relative abundance of greater than $10 \%$, the presence of 6 genera common to unused and used RMS samples was noticed as follows: Acinetobacter, Cellvibrio, Flavobacterium, Luteimonas, Pseudomonas, and Symbiobacterium. We found 495 genera common to unused and used RMS, 34 genera unique to unused RMS, and 81 genera unique to used RMS (Supplemental Table S6; https://github .com/CRSV).

The $\alpha$-diversity was lower in unused RMS compared with used RMS samples (observed: $P<0.001$; Shannon: $P=0.005$; InvSimpson: $P=0.012$; Figure $4 \mathrm{~A}$ ). Moreover, a difference was observed between unused and used RMS regarding the structure of the microbiota $(P<0.001$; Figure 4B). Listeria monocytogenes was detected in 30\% (95\% CI: 15-50) of unused RMS samples and in $48 \%$ (95\% CI: 29-68) of used RMS beddings. Salmonella spp. were found in 11\% (95\% CI: 3-30) of unused RMS samples and in 15\% (95\% CI: 5-35) of used RMS samples. Unused and used RMS bedding did not significantly differ regarding pathogen detection. Finally, higher counts of bacteria, in general, were noticed in used RMS samples compared with unused samples, with differences in magnitude ranging from $1.0 \log 10 \mathrm{cfu} / \mathrm{g}$ (for E. coli) to $1.6 \log 10 \mathrm{cfu} / \mathrm{g}$ (for Staphylococcus spp.; Table 3).

Housing type was included in all models comparing bacterial counts between RMS and straw bedding samples. Therefore, to better appraise the effect of that potential confounder, unconditional associations between housing type and bacterial counts are presented as supplementary materials in Supplemental Table S7 (https://github.com/CRSV). Moreover, unadjusted associations between bedding type and bacterial counts are also presented as supplementary materials in Supplemental Table S8 (https://github.com/CRSV). Briefly, adjusting or not for confounding by housing type of the bedding type-bacterial count associations had little effect on the computed least squares means estimates.

Influence of Method of Preparation on RMS Unused Bedding Microbiota. Finally, the microbiota in the samples originating from the unused RMS samples produced using different production systems were compared. Among the 27 RMS herds, 5 RMS production methods were observed as follows: separation followed by maturation in a rotating drum (S-CRD; $\mathrm{n}=2)$, anaerobic digestion $(\mathbf{A D} ; \mathrm{n}=1)$, separation followed by maturation in a heap $(\mathbf{S}-\mathbf{M H} ; \mathrm{n}=10)$, separation with maturation in an open or enclosed container $(\mathbf{S}-\mathbf{M B} ; \mathrm{n}=13)$, and separation only $(\mathbf{S} ; \mathrm{n}=1)$. Due to the low number of $\mathrm{S}-\mathrm{CRD}, \mathrm{AD}$, and $\mathrm{S}$ production systems, and the resulting low statistical power to highlight differences between systems, the analysis was carried out on the S-MH and S-MB production systems. The same phyla were shown in each production methods, with no significant difference in relative abundance (Figure 7A). Both S-BM and S-MH production systems were associated with a high proportion of Acinetobacter genus in RMS, and an overrepresentation of unclassified Bacillales appeared for S-MH (Figure $7 \mathrm{~B})$.

No significant differences in $\alpha$-diversity could be observed for any of the diversity indices between the 2 production groups (observed: $P=0.94$; Shannon: $P=$ 0.65; InvSimpson: $P=0.92$; Figure $8 \mathrm{~A}$ ). However, the microbiota structure was affected by the production method $(P<0.01$; Figure 8B).

When comparing genus composition of the different RMS production systems, we obtained a total of 804 genera. Genera not present in at least $90 \%$ of the samples (10 S-MH and $13 \mathrm{~S}-\mathrm{MB})$ were discarded. The core microbiota shared by bedding from the 2 production systems consisted of 275 genera, whereas 4 genera unique to the S-MB production system and 15 genera unique to the S-MH production system were found (Supplemental Table S9; https://github.com/CRSV).

Listeria monocytogenes was detected in 55\% (95\% CI: 25-82) of unused RMS samples produced using the S-MB system and in 20\% (95\% CI: 4-56) of samples produced using the S-MH system. Also, we observed that Salmonella spp. was detected in 100\% (95\% CI: 95-100\%) of unused RMS samples produced using the S-MB system compared with none (95\% CI: 0-34\%) in samples produced using the S-MH system.

Estimates of least squares means of bacterial counts in unused RMS bedding and as a function of production system are reported in Table 4. Briefly, streptococci and $E$. coli counts were higher in samples obtained using S-MB (streptococci: $5.7 \log 10 \mathrm{cfu} / \mathrm{g} ; 95 \% \mathrm{CI}$ : 5.1-6.4; E. coli: $4.9 \log 10 \mathrm{cfu} / \mathrm{g} ; 95 \%$ CI: 4.6-5.3) compared with S-MH (streptococci: $4.3 \log 10 \mathrm{cfu} / \mathrm{g} ; 95 \%$ CI: 3.5-5.0; E. coli: $4.2 \log 10 \mathrm{cfu} / \mathrm{g} ; 95 \%$ CI: 3.8-4.6; Table 4).

\section{DISCUSSION}

The use of RMS is an alternative to classical straw bedding for dairy cows. Multiple benefits of RMS have been reported, including economic, increased cow comfort, reduced hock lesions, and improved cleanliness of the animals (Green et al., 2014; Bradley et al., 2018; 
A RMS Phylum Composition depending on the production type

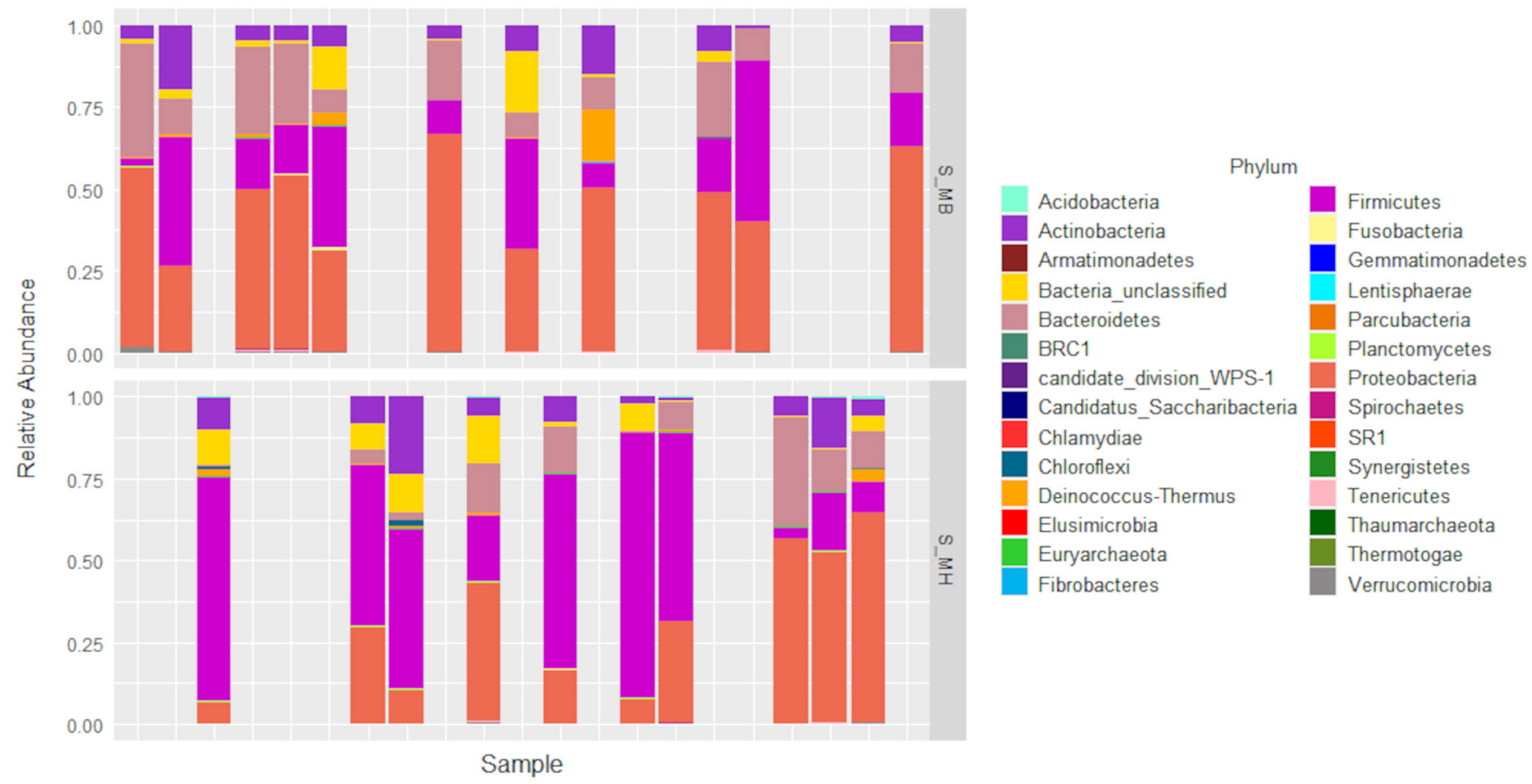

B RMS Composition depending on the production type

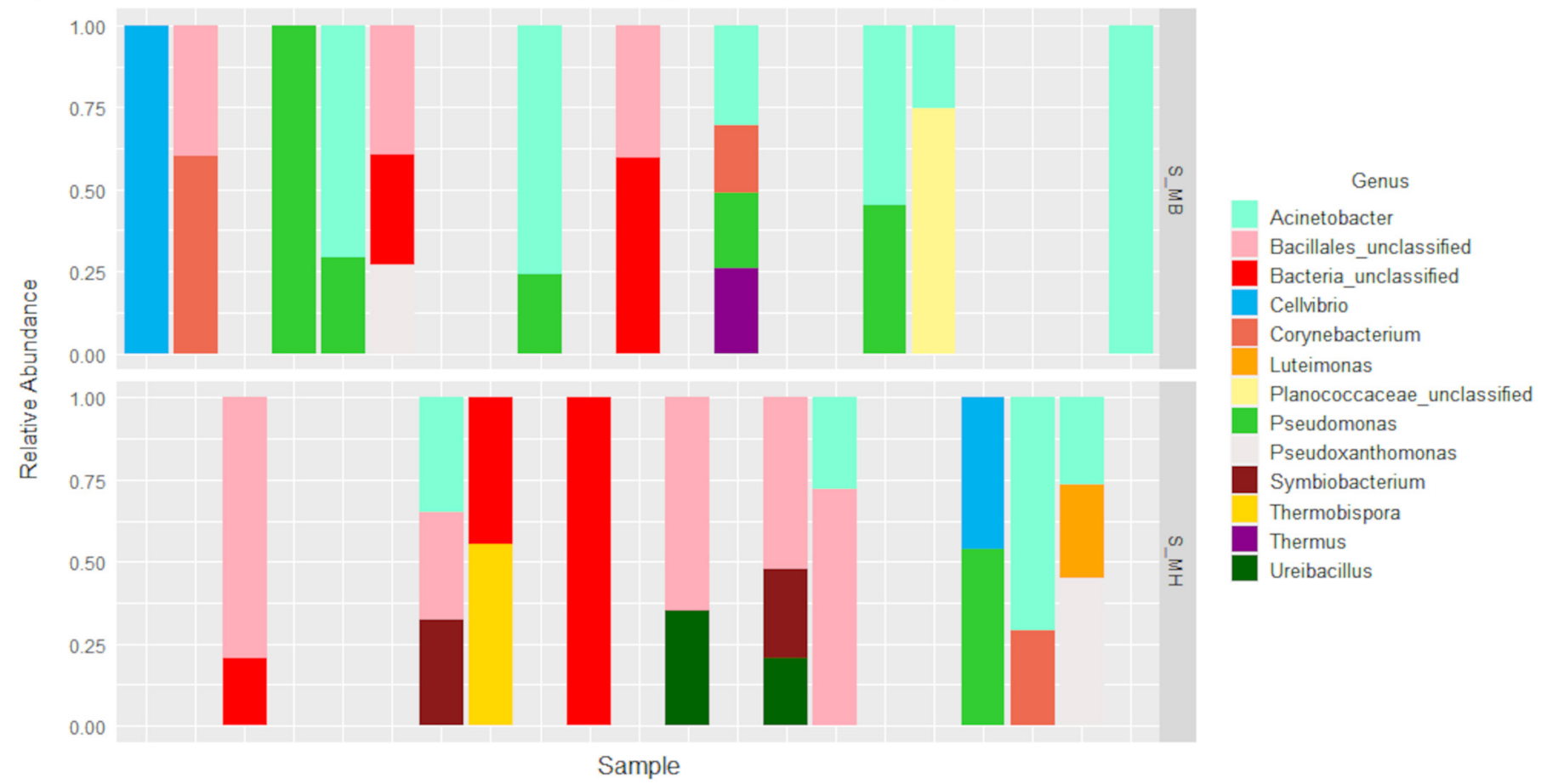

Figure 7. Phyla repartition in unused recycled manure solids (RMS) production. (A) Phyla retrieved from unused RMS bedding obtained from the main 2 types of RMS production were compared as follows: separation followed by maturation in a heap $(\mathrm{S}-\mathrm{MH} ; \mathrm{n}=10)$ and separation with maturation in an opened or enclosed container $(\mathrm{S}-\mathrm{MB} ; \mathrm{n}=11$ ). Abundance filter was set to zero, and 28 phyla were obtained. (B) Genera retrieved from unused RMS bedding obtained from the 2 main types of RMS production were compared as follows: $\mathrm{S}-\mathrm{MH}(\mathrm{n}=10)$ and $\mathrm{S}-\mathrm{MB}$ $(\mathrm{n}=11)$. The abundance filter was set to 0.10 , and 13 genera were obtained. 


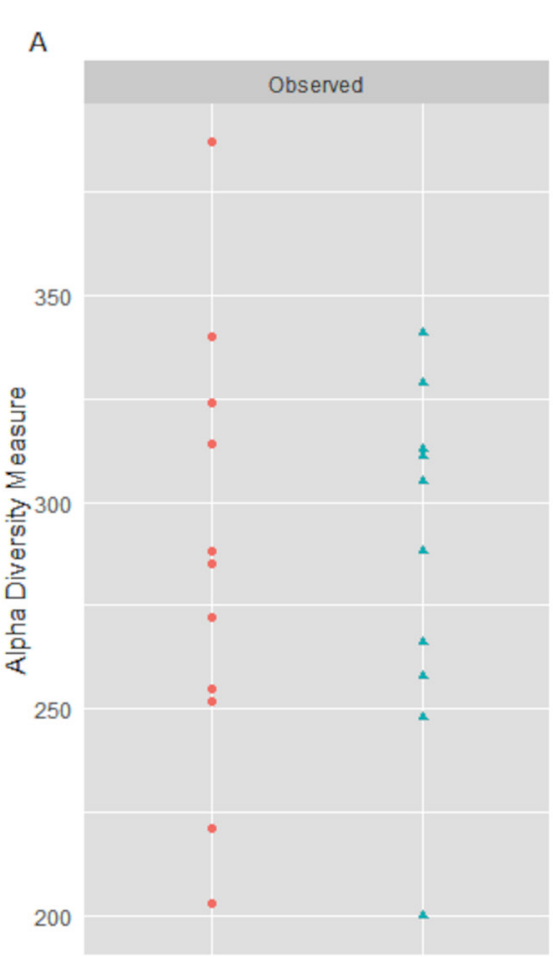

B

\section{Alpha diversity of RMS production}
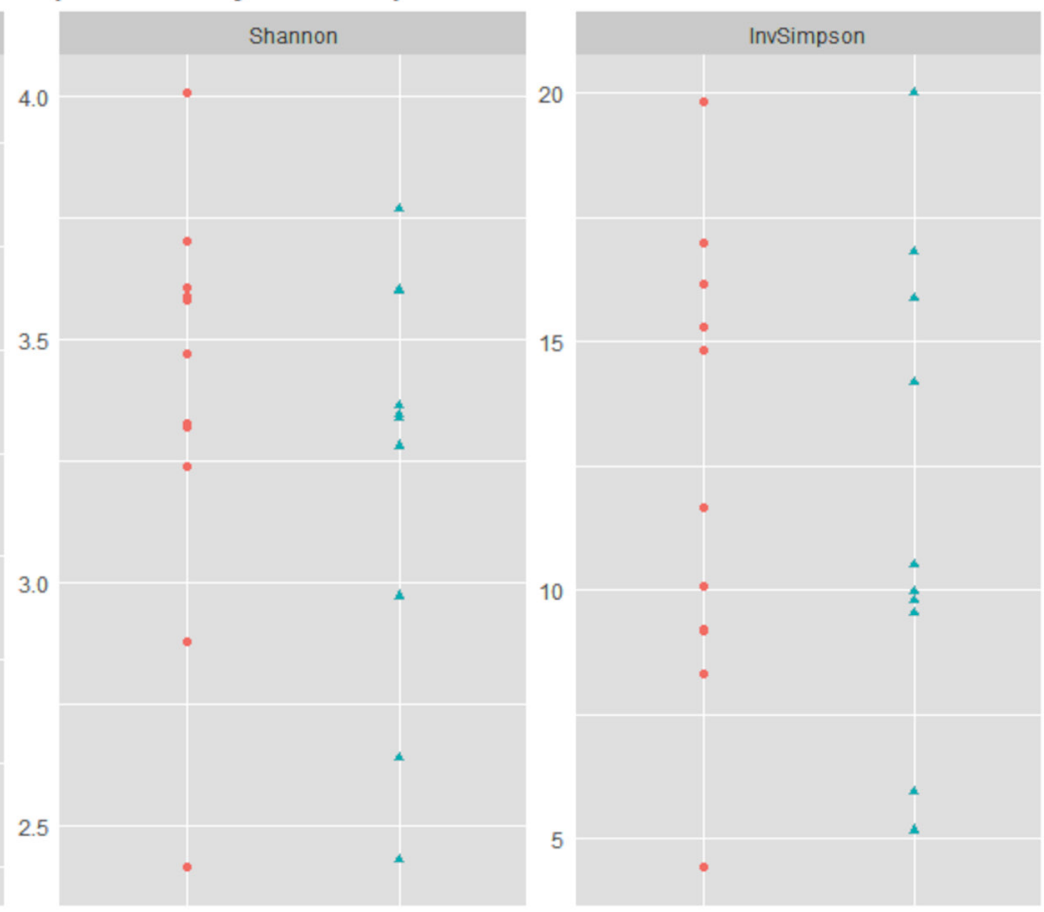

Production

- S_MB

A. S.MH

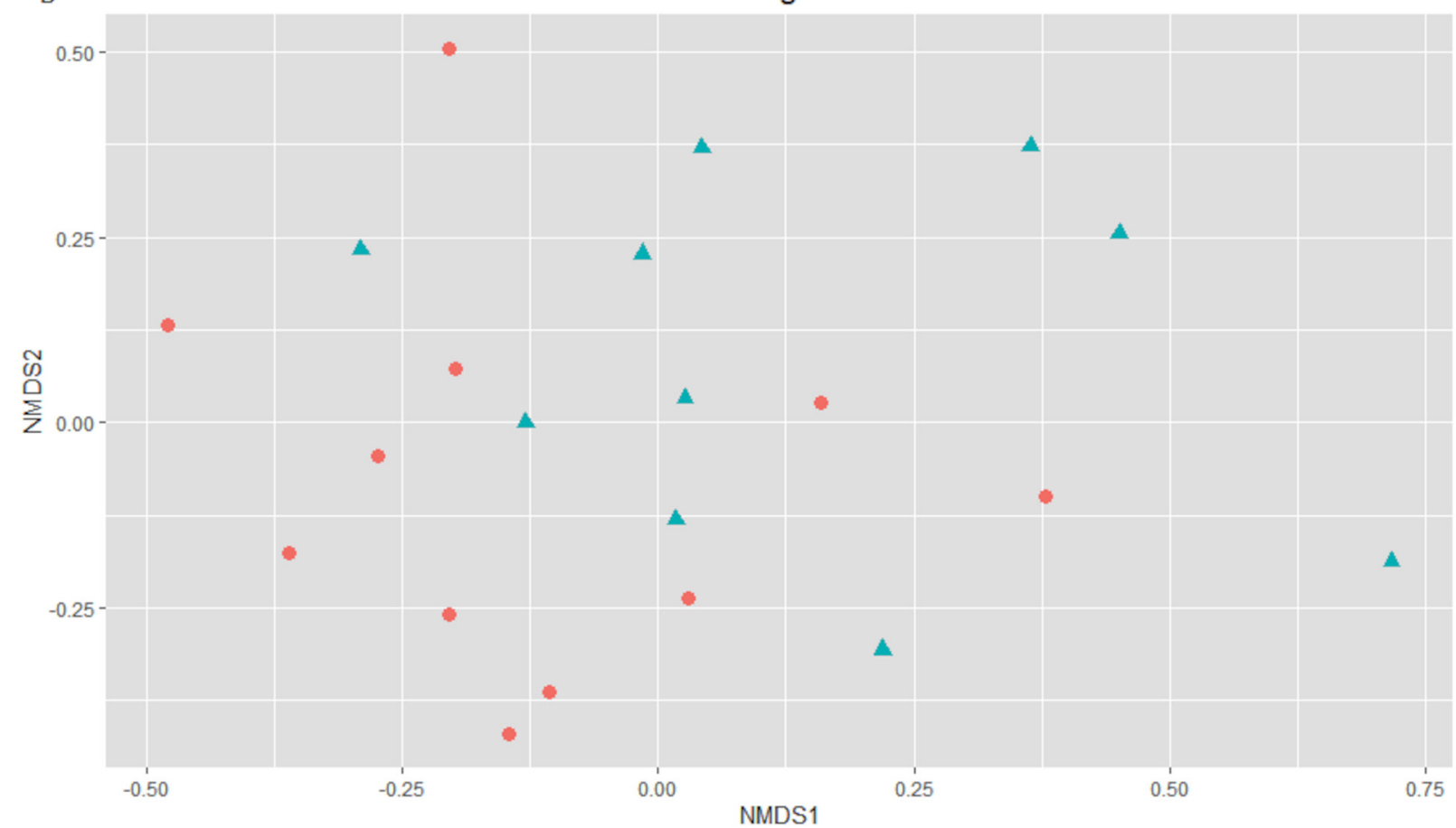

Production

- S_MB

A S_MH

Figure 8. Microbiota analysis of unused recycled manure solids (RMS) bedding obtained from the 2 main types of RMS production as follows: separation followed by maturation in a heap $(\mathrm{S}-\mathrm{MH} ; \mathrm{n}=10)$ and separation with maturation in an opened or enclosed container (S-MB; $\mathrm{n}$ =11). (A) The $\alpha$-diversity was measured by observed, Shannon, and InvSimpson indices. Statistical comparison was performed using KruskalWallis test $(P<0.05)$. (B) Nonmetric multidimensional scaling (NMDS) plot representing microbiota $\beta$-diversity. Each point represents a single unused RMS microbiota associated with one production system and was based on 861 operational taxonomic units. Jaccard and Bray-Curtis indices and ADONIS statistical tests $(P<0.05)$ were performed. 
Fournel et al., 2019b). However, no study has described the microbiota of used and unused RMS on dairy farms in Canada. Actually, they were very few studies describing microbiota of straw bedding.

The $\alpha$-diversity was higher in unused RMS as compared with unused straw, confirmed by a higher presence of genera unique to unused RMS compared with unused straw. The NMDS analyses illustrated a clear separated clustering of unused RMS and unused straw bedding samples. Those differences in diversity, both $\alpha$ and $\beta$, can be explained by the nature, the origin, and the physicochemical properties of the different bedding types. The microbiological quality of RMS bedding can be influenced by temperature, humidity, $\mathrm{pH}$, oxygento-nitrogen ratio, and by the on-farm production system (Hanajima et al., 2011; Pandey et al., 2018; Meng et al., 2019). The core microbiota should be similar from one region to another because the origin of RMS bedding is feces, but the fine microbiota composition should be region dependent as regional factors such as humidity, temperature, and feed composition will affect the microbiota (Hanajima et al., 2011; Rice et al., 2012; Pandey et al., 2018). Straw bedding microbiota can be influenced by geographical location, humidity, or separation methods (Djemiel et al., 2017; Tang et al., 2017).

Unused RMS consist of undigested plant fibers that are similar, to some degree, to straw (Godbout et al., 2019). Unused RMS and unused straw possessed mostly the same main phyla but in different proportions. However, before its use, the RMS already presented the phyla contained in manure to a much greater extent than did unused straw bedding. According to a study on the microbiota of dairy cows, Firmicutes and Bacteroidetes are the dominant phyla, and Proteobacteria and Actinobacteria are minor contributors to the microbiota of dairy cow feces (Hagey et al., 2019). In our study, we observed the same dominant phyla (Firmicutes and Bacteroidetes) in RMS bedding, but the proportion of Proteobacteria was increased compared with the study by Hagey et al. (2019). We hypothesized that the environmental and seasonal characteristics such as humidity and temperature affect Proteobacteria. Our study took place in eastern Canada during winter time. Farms studied in Hagey et al. (2019) were located in California.

The microbiota of unused straw was composed mainly of Proteobacteria, Bacteroidetes, Actinobacteria, and Firmicutes. These phyla were found in another study of straw microbiota but with different relative abundances (Liu et al., 2016). These differences could be explained by parameters related to straw origin, soil, or climate.

The microbiota of unused and used RMS were similar, as illustrated by the NMDS representation; this was striking when compared with unused and used straw. Unused RMS formed a different group from used RMS, but the 2 showed a large overlap compared with unused and used straw, which formed 2 distinct groups. This result was confirmed by the Venn analysis, where 495 OTU out of 610 were common to unused and used RMS, compared with 390 OTU out of 624 that were common to unused and used straw. These results illustrated that the microbiota of unused and used RMS were quite similar, and use (i.e., mainly urine and fecal contamination) did not have a major effect on microbiota diversity, which was the opposite of what was observed for straw. After use, RMS contaminated with feces or urine would be collected and the RMS production process run again (Leach et al., 2015; Fournel et al., 2019a,b). This process could be repeated indefinitely and could contribute to the stabilization of the microbial population found.

We detected equivalent bacterial count levels for total mesophilic bacteria, Staphylococcus spp., Streptococcus spp., coliforms, Klebsiella spp., and E. coli in unused RMS compared with those found in other studies. For used RMS bacterial count, our results were $1 \log 10$ $\mathrm{cfu} / \mathrm{g}$ higher than those reported elsewhere (Leach et al., 2015; Patel et al., 2019; Rowe et al., 2019). Different production practices and environmental conditions between Canada and the United States or the United Kingdom and the time when bedding was sampled (winter vs. summer; Okamoto et al., 2018) should be explored to explain these differences, but they do justify a regional analysis such as the one we conducted.

Recently, Lasprilla-Mantilla et al. (2019) demonstrated that Cryptosporidium spp., a zoonotic microorganism, was not affected by RMS production methods used on eastern Canadian dairy farms. Indeed, they found the bacterium in a higher proportion in RMS farms (85\%) compared with farms using straw (49\%) and suggested that the use of RMS could promote within-herd transmission. This suggests that other zoonotic pathogens could be present in RMS. Indeed, we detected the presence of Listeria monocytogenes and Salmonella spp. in unused RMS. It is important to mention that our results probably underestimated the actual prevalence of Salmonella spp., L. monocytogenes, and other possible human pathogens due to the limited diagnostic sensitivity of the tests used. (Kérouanton et al., 2010; BIO RAD, 2014; Lebel et al., 2017; Thibodeau et al., 2017). A recent study reported the presence of E. coli, Salmonella, and Campylobacter in the cow feces on several farms in the United States (Hagey et al., 2019), highlighting the need for an RMS maturation process that should completely eliminate these zoonotic pathogens, which was clearly not the case for the majority of 
RMS samples analyzed in our study. Several maturation processes exist, such as $\mathrm{AD}$, drum composting, closed container "in-vessel," and maturation in an opened or enclosed container or a heap. Maturation can increase temperature for maximal pathogen destruction, microbial stabilization, and moisture reduction (Fournel et al., 2019a). Enclosed and ventilated containers may be useful as they enable a more standardized control of environmental conditions, and can thus provide a uniform thermophilic treatment and year-round production of bedding with consistent quality. Chemical conditioners, such as alkaline conditioners, can reduce bacterial counts in recycled manure and can be another option for maturation (Hogan et al., 1999).

We also found that the type of RMS production system affected the presence of pathogens and microbiota structure. A study of dairy manure composting processes also observed that the different composting steps (active and maturation) can affect microbiota composition (Zhong et al., 2020). According to other recent articles, the farm origin, the farm housing type, the manure state, and the different composting phases can affect the bacterial communities in dairy manure (Pandey et al., 2018; Hagey et al., 2019; Zhong et al., 2020). In our study, heap-matured RMS samples showed lower bacterial counts than enclosed container-matured samples. According to the Ministry of Sustainable Development, Environment and the Fight Against Climate Change in Québec, standards for efficient pathogen destruction in compost are as follows: the compost needs to reach $55^{\circ} \mathrm{C}$ or more for at least $3 \mathrm{~d}$ in the active composting phase and needs to be turned frequently for proper oxygen introduction (Misra et al., 2005; Bernal et al., 2009; Ministère du Développement Durable, 2018). These criteria could possibly be implemented as critical control points for the on-farm production of RMS for improving composting and maturation technic. On the other hand, ensuring that a certain temperature is reached could lead to the selection of thermoresistant bacterial populations. These latter microorganisms could potentially affect the production of various dairy products (Gagnon et al., 2020).

An important strength of our study was investigating RMS bedding as it is currently produced on dairy farms, using an observational cross-sectional study design. As a result, this study design also led to an important study limitation, the potential introduction of a confounding bias. In our case, our estimates seemed to be unaffected by one of the potentially most important confounders, housing type. Nevertheless, other unmeasured confounders were possibly operating and could have biased our estimates. In the future, an experimental study design would be needed to confirm our results.

\section{CONCLUSIONS}

The microbiota of RMS differed from that of straw, and the method for producing the RMS clearly affected its quality. In our study, RMS heap production appeared to be a better option for RMS maturation than production using maturation in an opened or enclosed container because it resulted in a lower bacterial load, and Listeria monocytogenes and Salmonella spp. were less frequently isolated. However, this would have to be confirmed in a study designed specifically for comparing production methods. Finally, this study clearly indicated that the microbial quality of RMS, in general, is inferior to straw bedding, the most common bedding used in eastern Canada. The RMS bedding currently produced on farms in eastern Canada clearly constitutes a greater microbiological risk for the dairy herds compared with straw bedding.

\section{ACKNOWLEDGMENTS}

This project was funded by the Conseil de Recherche en Sciences Naturelles et en Génie du Canada (CRSNG; Ottawa, Canada; grant \#CRDPJ-499421-16), the Fonds de Recherche du Québec-Nature et Technologies (FQRNT; Québec, Canada; grant \#2017-LG-201835), the Consortium de Recherche et Innovations en Bioprocédés Industriels au Québec (CRIBIQ; Québec, Canada; grant \#2015-044-C17), and Novalait Inc. (Brainville-sur-Meuse, Canada; grant \#RB002525). The English language was reviewed and corrected by Proof-Reading-Service.com (Devonshire Business Center, Letchworth Garden City, Hertfordshire, UK). The authors thank the Op+Lait research group (SaintHyacinthe, Canada) for the exchange of ideas that led to the conception of this project, Caroline Forest (Faculté de Médecine Vétérinaire, Université de Montréal, Saint-Hyacinthe, Canada) for the farm visits and bedding samples, and Génome Québec (Montréal, Canada) for sample sequencing. Special thanks are due to all members of the CRSV (Saint-Hyacinthe, Canada) team for their scientific advice and technical assistance. The authors have not stated any conflicts of interest.

\section{REFERENCES}

Animal and Health Diagnostic Center. 2020. How to collect bedding samples. Cornell University, College of Veterinary Medicine.

Bernal, M. P., J. A. Alburquerque, and R. Moral. 2009. Composting of animal manures and chemical criteria for compost maturity assessment. A review. Bioresour. Technol. 100:5444-5453. https:// doi.org/10.1016/j.biortech.2008.11.027.

Bio-Rad. 2014. RAPID'L.mono/Agar User Guide. Pages 1-7. Vol. 2. Bio-Rad Laboratories. Accessed Oct. 6, 2021. https://www.bio -rad.com/sites/default/files/webroot/web/pdf/fsd/literature/ 10000127436.pdf. 
Bradley, A. J., K. A. Leach, M. J. Green, J. Gibbons, I. C. Ohnstad, D. H. Black, B. Payne, V. E. Prout, and J. E. Breen. 2018. The impact of dairy cows' bedding material and its microbial content on the quality and safety of milk - A cross sectional study of UK farms. Int. J. Food Microbiol. 269:36-45. https://doi.org/10.1016/ j.ijfoodmicro.2017.12.022.

Caporaso, J. G., C. L. Lauber, W. A. Walters, D. Berg-Lyons, J. Huntley, N. Fierer, S. M. Owens, J. Betley, L. Fraser, M. Bauer, N. Gormley, J. A. Gilbert, G. Smith, and R. Knight. 2012. Ultrahigh-throughput microbial community analysis on the Illumina HiSeq and MiSeq platforms. ISME J. 6:1621-1624. https://doi .org/10.1038/ismej.2012.8.

Carroll, E. J., and D. E. Jasper. 1978. Distribution of Enterobacteriaceae in recycled manure bedding on California dairies. J. Dairy Sci. 61:1498-1508. https://doi.org/10.3168/jds.S0022-0302(78)83754 -0 .

Cole, K. J., and J. S. Hogan. 2016. Short communication: Environmental mastitis pathogen counts in freestalls bedded with composted and fresh recycled manure solids. J. Dairy Sci. 99:1501-1505. https://doi.org/10.3168/jds.2015-10238.

Djemiel, C., S. Grec, and S. Hawkins. 2017. Characterization of bacterial and fungal community dynamics by high-throughput sequencing (HTS) metabarcoding during flax dew-retting. Front. Microbiol. 8:2052. https://doi.org/10.3389/fmicb.2017.02052.

Dohoo, I., W. Martin, and H. Stryhn. 2009. Pages 339-340 in Veterinary Epidemiologic Research, 2nd ed. AVC Inc.

Dufour, S. 2021. Classical microbiology data - Beauchemin et al., 2021. Scholars Portal Dataverse, V1. https://doi.org/10.5683/ SP2/8HRWGR.

Dufour, S., H. W. Barkema, L. DesCôteaux, T. J. DeVries, I. R. Dohoo, K. Reyher, J.-P. Roy, and D. T. Scholl. 2010. Development and validation of a bilingual questionnaire for measuring udder health related management practices on dairy farms. Prev. Vet. Med. 95:74-85. https://doi.org/10.1016/j.prevetmed.2010.02.018.

Fournel, S., S. Godbout, P. Ruel, A. Fortin, K. Duquette-Lozeau, V. Létourneau, M. Généreux, J. Lemieux, D. Potvin, C. Côté, C. Duchaine, and D. Pellerin. 2019a. Production of recycled manure solids for use as bedding in Canadian dairy farms: II. Composting methods. J. Dairy Sci. 102:1847-1865. https://doi.org/10.3168/jds .2018-14967.

Fournel, S., S. Godbout, P. Ruel, A. Fortin, M. Généreux, C. Côté, C. Landry, and D. Pellerin. 2019b. Production of recycled manure solids for bedding in Canadian dairy farms: I. Solid-liquid separation. J. Dairy Sci. 102:1832-1846. https://doi.org/10.3168/jds .2018-14966.

Gagnon, M., L. Hamelin, A. Fréchette, S. Dufour, and D. Roy. 2020. Effect of recycled manure solids as bedding on bulk tank milk and implications for cheese microbiological quality. J. Dairy Sci. 103:128-140. https://doi.org/10.3168/jds.2019-16812.

Godbout, S., S. Adam, L. Belzile, P. Brassard, L. Chauveau, C. Côté, C. Duchaine, S. Dufour, K. Duquette-Lozeau, G. Fecteau, S. Fournel, M. Généreux, D. Godonou, C. Landry, V. Létourneau, A. Lévesque, S. Ouslati, D. Potvin, P. Ruel, and M. Villettaz-Robichaud. 2019. Litière recyclée en production laitière - État des connaissances et balises. I. d. r. e. d. d. e. a. Centre de recherche en sciences animales de Deschambault, Université Laval, ed. Centre de recherche en sciences animales de Deschambault, Institut de recherche et de développement en agroenvironnement, Université Laval, Québec.

Godden, S., R. Bey, K. Lorch, R. Farnsworth, and P. Rapnicki. 2008. Ability of organic and inorganic bedding materials to promote growth of environmental bacteria. J. Dairy Sci. 91:151-159. https: //doi.org/10.3168/jds.2007-0415.

Green, M., K. A. Leach, J. Breen, I. Ohnstad, S. Tuer, S. Archer, and A. Bradley. 2014. Recycled manure solids as bedding for dairy cattle: A scoping study. Cattle Pract. 22:207-214.

Hagey, J. V., S. Bhatnagar, J. M. Heguy, B. M. Karle, P. L. Price, D. Meyer, and E. A. Maga. 2019. Fecal microbial communities in a large representative cohort of California dairy cows. Front. Microbiol. 10:1093. https://doi.org/10.3389/fmicb.2019.01093.
Hanajima, D., Y. Fukumoto, T. Yasuda, K. Suzuki, K. Maeda, and R. Morioka. 2011. Bacterial community dynamics in aerated cow manure slurry at different aeration intensities. J. Appl. Microbiol. 111:1416-1425. https://doi.org/10.1111/j.1365-2672.2011.05151.x.

Hogan, J. S., V. L. Bogacz, L. M. Thompson, S. Romig, P. S. Schoenberger, W. P. Weiss, and K. L. Smith. 1999. Bacterial counts associated with sawdust and recycled manure bedding treated with commercial conditioners. J. Dairy Sci. 82:1690-1695. https://doi .org/10.3168/jds.S0022-0302(99)75398-1.

Holschbach, C. L., and S. F. Peek. 2018. Salmonella in dairy cattle. Vet. Clin. North Am. Food Anim. Pract. 34:133-154. https://doi .org/10.1016/j.cvfa.2017.10.005.

Hunt, K., N. Drummond, M. Murphy, F. Butler, J. Buckley, and K. Jordan. 2012. A case of bovine raw milk contamination with Listeria monocytogenes. Ir. Vet. J. 65:13. https://doi.org/10.1186/ 2046-0481-65-13.

Kérouanton, A., M. Marault, L. Petit, J. Grout, T. T. Dao, and A. Brisabois. 2010. Evaluation of a multiplex PCR assay as an alternative method for Listeria monocytogenes serotyping. J. Microbiol. Methods 80:134-137. https://doi.org/10.1016/j.mimet.2009 .11 .008 .

Kozich, J. J., S. L. Westcott, N. T. Baxter, S. K. Highlander, and P. D. Schloss. 2013. Development of a dual-index sequencing strategy and curation pipeline for analyzing amplicon sequence data on the MiSeq Illumina sequencing platform. Appl. Environ. Microbiol. 79:5112-5120. https://doi.org/10.1128/AEM.01043-13.

Larivière-Gauthier, G., A. Thibodeau, A. Letellier, E. Yergeau, and P. Fravalo. 2017. Reduction of Salmonella shedding by sows during gestation in relation to its fecal microbiome. Front. Microbiol. 8:2219. https://doi.org/10.3389/fmicb.2017.02219.

Lasprilla-Mantilla, M. I., V. Wagner, J. Pena, A. Frechette, K. Thivierge, S. Dufour, and C. Fernandez-Prada. 2019. Effects of recycled manure solids bedding on the spread of gastrointestinal parasites in the environment of dairies and milk. J. Dairy Sci. 102:1130811316. https://doi.org/10.3168/jds.2019-16866.

Leach, K. A., S. C. Archer, J. E. Breen, M. J. Green, I. C. Ohnstad, S. Tuer, and A. J. Bradley. 2015. Recycling manure as cow bedding: Potential benefits and risks for UK dairy farms. Vet. J. 206:123130. https://doi.org/10.1016/j.tvjl.2015.08.013.

Lebel, P., A. Letellier, J. Longpré, B. Laplante, E. Yergeau, and P. Fravalo. 2017. Feed presentation options in Swine early fattening mitigates Salmonella shedding and specifically modulates the faecal microbiota. J. Appl. Microbiol. 122:30-39. https://doi.org/10 $.1111 /$ jam.13305.

Liu, J., M. Zhang, C. Xue, W. Zhu, and S. Mao. 2016. Characterization and comparison of the temporal dynamics of ruminal bacterial microbiota colonizing rice straw and alfalfa hay within ruminants. J. Dairy Sci. 99:9668-9681. https://doi.org/10.3168/jds .2016-11398.

Meng, Q., W. Yang, M. Men, A. Bello, X. Xu, B. Xu, L. Deng, X. Jiang, S. Sheng, X. Wu, Y. Han, and H. Zhu. 2019. Microbial community succession and response to environmental variables during cow manure and corn straw composting. Front. Microbiol. 10:529. https://doi.org/10.3389/fmicb.2019.00529.

Ministère du Développement Durable de l'Environnement et des Parcs. 2018. Lignes directrices pour l'encadrement des activités de compostage. Gouvernement du Québec, ed. Gouvernement du Québec, Québec. https://ocpm.qc.ca/sites/ocpm.qc.ca/files/pdf/ P61/5a.pdf.

Misra, R. V., R. N. Roy, and H. Hiraoka. 2005. Méthode de compostage au niveau de l'exploitation agricole. Pages 3-32 in Documents de Travail sur les Terres et les Eaux. Organisation des Nations Unies pour l'Alimentation et l'Agriculture, ed. Food and Agriculture Organisation (FAO).

Nightingale, K. K., E. D. Fortes, A. J. Ho, Y. H. Schukken, Y. T. Grohn, and M. Wiedmann. 2005. Evaluation of farm management practices as risk factors for clinical listeriosis and fecal shedding of Listeria monocytogenes in ruminants. J. Am. Vet. Med. Assoc. 227:1808-1814. https://doi.org/10.2460/javma.2005.227.1808.

Okamoto, E., H. Miyanishi, A. Nakamura, T. Kobayashi, N. Kobayashi, Y. Terawaki, and H. Nagahata. 2018. Bacteriological evaluation 
of composted manure solids prepared from anaerobic digested slurry for hygienic recycled bedding materials for dairy cows. Anim. Sci. J. 89:727-732. https://doi.org/10.1111/asj.12962.

Organisation Mondiale de la Santé animale (OIE). 2019. Prévention et Maîtrise des Salmonelles dans les Systèmes de Production Commerciale de Bovins. Accessed Apr. 10, 2020. https://www.oie.int/ $\mathrm{fr} /$ ce-que-nous-faisons/normes/codes-et-manuels/acces-en-ligne -au-code-terrestre $/$ id $=169 \& \mathrm{~L}=1 \&$ htmfile=chapitre_salmonella pig.htm.

Pandey, P., C. Chiu, M. Miao, Y. Wang, M. Settles, N. S. del Rio, A. Castillo, A. Souza, R. Pereira, and R. Jeannotte. 2018. 16S rRNA analysis of diversity of manure microbial community in dairy farm environment. PLoS One 13:e0190126. https://doi.org/10.1371/ journal.pone.0190126.

Patel, K., S. M. Godden, E. Royster, B. A. Crooker, J. Timmerman, and L. Fox. 2019. Relationships among bedding materials, bedding bacteria counts, udder hygiene, milk quality, and udder health in US dairy herds. J. Dairy Sci. 102:10213-10234. https://doi.org/10 .3168/jds.2019-16692.

Rice, W. C., M. L. Galyean, S. B. Cox, S. E. Dowd, and N. A. Cole. 2012. Influence of wet distillers grains diets on beef cattle fecal bacterial community structure. BMC Microbiol. 12:25. https://doi .org/10.1186/1471-2180-12-25.

Robles, I., D. F. Kelton, H. W. Barkema, G. P. Keefe, J. P. Roy, M. A. G. von Keyserlingk, and T. J. DeVries. 2020. Bacterial concentrations in bedding and their association with dairy cow hygiene and milk quality. Animal 14:1052-1066. https://doi.org/10.1017/ S1751731119002787.

Rowbotham, R. F., and P. L. Ruegg. 2016a. Associations of selected bedding types with incidence rates of subclinical and clinical mastitis in primiparous Holstein dairy cows. J. Dairy Sci. 99:47074717. https://doi.org/10.3168/jds.2015-10675.

Rowbotham, R. F., and P. L. Ruegg. 2016b. Bacterial counts on teat skin and in new sand, recycled sand, and recycled manure solids used as bedding in freestalls. J. Dairy Sci. 99:6594-6608. https:// doi.org/10.3168/jds.2015-10674.

Rowe, S. M., S. M. Godden, E. Royster, J. Timmerman, B. A. Crooker, and M. Boyle. 2019. Cross-sectional study of the relationships among bedding materials, bedding bacteria counts, and intramammary infection in late-lactation dairy cows. J. Dairy Sci. 102:11384-11400. https://doi.org/10.3168/jds.2019-17074.

Tang, M. T., H. Han, Z. Yu, T. Tsuruta, and N. Nishino. 2017. Variability, stability, and resilience of fecal microbiota in dairy cows fed whole crop corn silage. Appl. Microbiol. Biotechnol. 101:63556364. https://doi.org/10.1007/s00253-017-8348-8.

Thibodeau, A., A. Letellier, É. Yergeau, G. Larrivière-Gauthier, and P. Fravalo. 2017. Lack of evidence that selenium-yeast improves chicken health and modulates the caecal microbiota in the context of colonization by Campylobacter jejuni. Front. Microbiol. 8. https: //doi.org/10.3389/fmicb.2017.00451.

Zhong, X. Z., X. Li, Y. Zeng, S. Wang, Z.-Y. Sun, and Y.-Q. Tang. 2020. Dynamic change of bacterial community during dairy manure composting process revealed by high-throughput sequencing and advanced bioinformatics tools. Bioresour. Technol. 306:123091. https://doi.org/10.1016/j.biortech.2020.123091.

\section{ORCIDS}

Jessika Beauchemin $\odot$ https://orcid.org/0000-0002-5542-2556 Annie Fréchette @ https://orcid.org/0000-0001-9363-3971 William Thériault (1) https://orcid.org/0000-0001-7546-4262 Simon Dufour ๑ https://orcid.org/0000-0001-6418-0424 Philippe Fravalo (ㄷ https://orcid.org/0000-0003-1796-0852

Alexandre Thibodeau $\odot$ https://orcid.org/0000-0003-1946-9143 